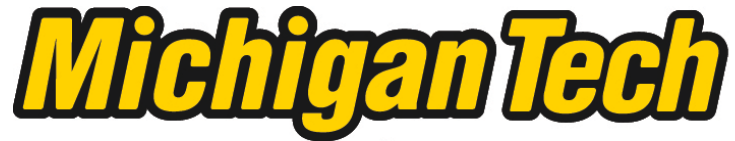 \\ Michigan Technological University Create the Future Digital Commons @ Michigan Tech
}

2014

PARTITIONING THE BLOCKS OF A STEINER TRIPLE SYSTEM INTO PARTIAL PARALLEL CLASSES

Jezerca Hodaj

Michigan Technological University

Follow this and additional works at: https://digitalcommons.mtu.edu/etds

Part of the Mathematics Commons

Copyright 2014 Jezerca Hodaj

\section{Recommended Citation}

Hodaj, Jezerca, "PARTITIONING THE BLOCKS OF A STEINER TRIPLE SYSTEM INTO PARTIAL PARALLEL CLASSES", Master's report, Michigan Technological University, 2014.

https://doi.org/10.37099/mtu.dc.etds/813

Follow this and additional works at: https://digitalcommons.mtu.edu/etds

Part of the Mathematics Commons 


\title{
PARTITIONING THE BLOCKS OF A STEINER TRIPLE SYSTEM INTO PARTIAL PARALLEL CLASSES
}

\author{
By \\ Jezerca Hodaj
}

\begin{abstract}
A REPORT
Submitted in partial fulfillment of the requirements for the degree of MASTER OF SCIENCES

in Mathematical Sciences
\end{abstract}

MICHIGAN TECHNOLOGICAL UNIVERSITY

2014

C2014 Jezerca Hodaj 

This report has been approved in partial fulfillment of the requirements for the Degree of MASTER OF SCIENCE in Mathematical Sciences.

Department of Mathematical Sciences

Report Advisor: Dr. Melissa Keranen

Committee Member: Dr. Donald Kreher

Committee Member: Dr. Nilufer Onder

Department Chair: Dr. Mark Gockenbach 

To the people I love the most in this world, my parents, who never stopped being on my side even though they were miles away. 


\section{Contents}

Contents vii

List of Figures viii

\begin{tabular}{|ll}
\hline Acknowledgments & 1
\end{tabular}

\begin{tabular}{|ll}
\hline Abstract & 3
\end{tabular}

$1 \quad$ About Coloring triples in every way 5

1.1 Introduction . . . . . . . . . . . . . . . . 5

1.2 Preliminary Results . . . . . . . . . . . . . . . . . . . 10

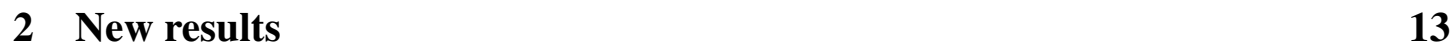

$2.1 \quad$ Dismantling Partial Parallel Classes . . . . . . . . . . . . . . . . . 13

2.2 New Results . . . . . . . . . . . . . . . . . . . . . . 15

$2.2 .1 \quad$ Partial Parallel classes of size $m=2 \ldots \ldots$. . . . . . 15

2.2.2 Partial parallel classes of size $m=4$. . . . . . . . . . 17

2.3 Special Cases . . . . . . . . . . . . . . . . 20

$2.3 .1 \quad$ When $v=21$ and $m=5 \ldots \ldots 20$

2.3 .2 When $v=33$ and $m=8 \ldots \ldots \ldots 24$

\begin{tabular}{|lll}
3 & Summary, Conclusions and Further Research & 37
\end{tabular}

3.1 Summary and Conclusions . . . . . . . . . . . . . . . . 37

3.2 Future work . . . . . . . . . . . . . . . . . 38

\begin{tabular}{|ll}
\hline Bibliography & 43
\end{tabular} 


\section{List of Figures}

$1.1 \quad$ STS(7), The Fano Plane $\ldots \ldots \ldots \ldots$

$2.1 \quad$ Partial parallel classes of size $5 \ldots \ldots \ldots \ldots$

2.2 Partial parallel classes of size $5 \ldots \ldots \ldots \ldots . \ldots \ldots$

2.3 Partial parallel classes of size $5 \ldots \ldots \ldots \ldots$

2.4 Partial parallel classes of size $5 \ldots \ldots \ldots \ldots \ldots$

2.5 Partial parallel classes of size $5 \ldots \ldots \ldots \ldots \ldots$

$2.6 \quad 3$-frame of type $2^{4}$ and an $R G D D\left(4^{3}\right)$. . . . . . . . . . . . . 30

2.7 Two 8 -PPC's using FPC1 . . . . . . . . . . . . . . . . . . . . 31

2.8 Two 8 -PPC's using FPC2 $\ldots \ldots \ldots \ldots \ldots \ldots \ldots$

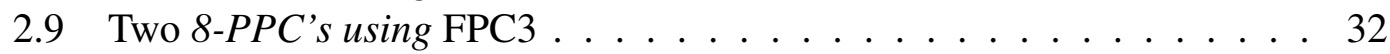

2.10 Two 8 -PPC's using FPC4 . . . . . . . . . . . . . . . . . 32

2.11 The $8-P P C$ created by all the blocks that we took out from the RGDD's. 33

2.12 Two 8 -PPC's using FPC1 . . . . . . . . . . . . . . . . . . . 34

2.13 Two 8 -PPC's using FPC2 . . . . . . . . . . . . . . . . . . 34

2.14 Two 8 -PPC's using FPC3 . . . . . . . . . . . . . . . . . . . . 35

2.15 Two 8 -PPC's using FPC4 . . . . . . . . . . . . . . . . . . . 35

2.16 The $8-P P C$ created by all the blocks that we took out from the RGDD's. 36 


\section{Acknowledgments}

I would like to express my great appreciation to Dr. Melissa Keranen. It's because of her guidance, her encouragement, and useful critiques in this research work that I am here today. She is one of the best professors I have had so far.

I would also like to deeply thank Dr. Don Kreher for all his help during these two years, and Dr. Nilufer Onder for accepting to be part of my graduate committee, and for all their advice.

In particular I would like to offer my thanks and gratitude to the Albanian Professor, Dr. Tanush Shaska. He is the person who opened the doors of this new world for me, and the reason I am here.

I would like to express my gratitude to all the professors that contributed on my path to this day. Among all of them, Dr. Tonchev, Stefaan De Winter, Ann Humes and Todd King. I would also like to thank Dr. Gockenbach for all his support.

A special thank you goes for my family. For my parents, my brother and my sisters. Thank you for all your unconditional love and support, for always believing in me. Thank you mostly for all the sacrifices that you have done for me all these years. I hope you will feel somehow proud of me.

Special thanks to my nieces and to my nephew. Your love is my optimism and my strength.

An important part of this achievement are my friends. The friends I left back home, and the friends I found here.

A special thank you to my friend Diego Domenzain. Thank you for your support, for your advice, and for always encouraging me to work harder! I really appreciate it!

Thank you my dear Anisa for your support. You were always there for me.

Andrew Lund - It was always nice to hear "You can do it Jes"!

Naima Khan, Moisés Hernandéz and Anirudh Balram - Thank you for being the friends that anyone needs!

Thank you all for being my family here!

I would like to offer my special thanks to Margaret Perander, Tory Connors, and Jeanne Meyers for being there every time that we needed your help. 


\section{Abstract}

Does there exist a Steiner Triple System on $v$ points, whose blocks can be partitioned into partial parallel classes of size $m$, where $m \leq\left\lfloor\frac{v}{3}\right\rfloor, m \mid b$ and $b$ is the number of blocks of the $S T S(v)$ ? We give the answer for $9 \leq v \leq 43$. We also show that whenever $2 \mid b, v \equiv 3(\bmod 6)$ we can find an $S T S(v)$ whose blocks can be partitioned into partial parallel classes of size 2 , and whenever $4 \mid b, v \equiv 3(\bmod 6)$, there exists an $S T S(v)$ whose blocks can be partitioned into partial parallel classes of size 4 . 


\section{Chapter 1}

\section{About Coloring triples in every way}

\subsection{Introduction}

A Steiner triple system on $v$ points, denoted as $S T S(v)$ is a pair $(V, \mathcal{B})$, where $V$ denotes a set of $v$ elements and $\mathcal{B}$ denotes a set of $b$ triples, called blocks, from $V$, such that $|\{B \in \mathcal{B}:\{x, y\} \subset B\}|=1$, for each distinct pair $\{x, y\} \subset V$. Each point of the Steiner triple system is contained in exactly $r=\frac{v-1}{2}$ blocks. Kirkman [7], showed the existence of such a system whenever the necessary conditions are met, in 1847 .

Theorem 1 [7] A Steiner triple system of order $v$ exists if and only if $v \equiv 1,3(\bmod 6)$.

The smallest Steiner triple system is $S T S(7)$, and is commonly known as the Fano Plane. The six lines and one circle drawn in Figure 1.1 gives a pictorial representation of the Steiner triple system with blocks;

$\mathcal{B}=\{\{1,4,3\},\{3,5,7\},\{1,5,2\},\{1,6,7\},\{2,3,6\},\{2,4,7\},\{4,5,6\}\}$. A parallel class in a Steiner triple system is a collection of disjoint blocks that partition its point set $V$. Because each point is in $r$ blocks we must have $r$ parallel classes, each consisting of $\frac{v}{3}$ blocks.

A Kirkman triple system on $v$ points, denoted as $K T S(v)$ is an $S T S(v)$ whose blocks can be partitioned into parallel classes.

Example 1.1.1 A Kirkman triple system of order 9

$\begin{array}{llllllllllll}1 & 2 & 3 & 1 & 4 & 7 & 1 & 5 & 9 & 1 & 6 & 8 \\ 4 & 5 & 6 & 2 & 5 & 8 & 2 & 6 & 7 & 2 & 4 & 9 \\ 7 & 8 & 9 & 3 & 6 & 9 & 3 & 4 & 8 & 3 & 5 & 7\end{array}$




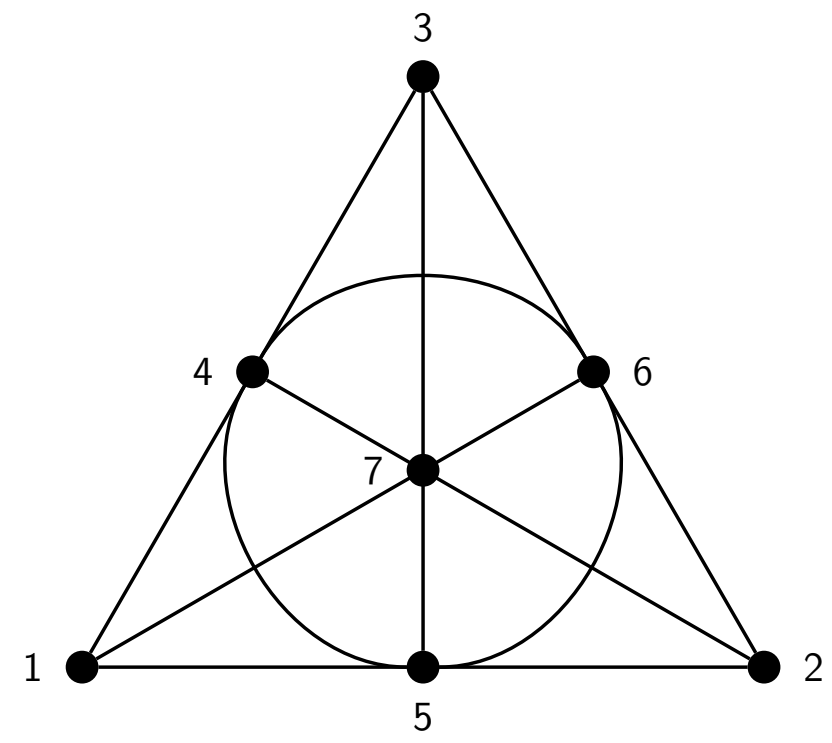

Figure 1.1: STS(7), The Fano Plane

Example 1.1.1 gives the 12 blocks of a $K T S(9)$. The blocks are partitioned into four parallel classes, and each parallel class partitions the set of nine points.

Theorem 2 [4 5. 7] A Kirkman triple system of order $v$ exists if and only if $v \equiv 3$ $(\bmod 6)$.

We are providing now more formal definitions.

A partial Steiner triple system on $v$ points, $\operatorname{PSTS}(\mathrm{v})$, is a pair $(V, \mathcal{B})$, where $V$ denotes a set of $v$ elements and $\mathcal{B}$ denotes a set of $b$ triples from $V$, such that $\left|B \cap B^{\prime}\right| \leq 1$, when $B, B^{\prime} \in \mathcal{B}$ and $B \neq B^{\prime}$.

When $\mathcal{R} \subseteq \mathcal{B}$ and $\left|B \cap B^{\prime}\right|=0$ when $B, B^{\prime} \in \mathcal{R}$ and $B \neq B^{\prime}$, then $\mathcal{R}$ is called a partial parallel class $(\mathrm{PPC})$ of $(V, \mathcal{B})$.

A partial parallel class containing all but one point of the points of a Steiner triple system, is called an almost parallel class.

A block coloring of a PSTS $(\mathrm{v})(\mathrm{V}, \mathcal{B})$ in $c$ colors is a mapping $\chi: \mathcal{B} \rightarrow\{1, \ldots, c\}$, so that every color class $\chi^{-1}(i), i \in\{1, \ldots, c\}$ is a partial parallel class.

The color type of a block coloring of $(\mathrm{V}, \mathcal{B})$ in $c$ colors is the sequence $\left(m_{1}, \ldots, m_{c}\right)$ 
where $m_{i}=|\{B \in \mathcal{B}: \chi(B)=i\}|$.

Often the sequence $\left(m_{1}, \ldots, m_{c}\right)$ is written in exponential form; color type $w_{1}^{u_{1}} \ldots w_{s}^{u_{s}}$ indicates that $u_{i}$ of the color classes have size $w_{i}$ for $1 \leq i \leq s$.

Using partial parallel classes we can give the definition of a Hanani triple system.

A Hanani triple system, (HATS) on $v=6 t+1$ points is a Steiner triple system having $\frac{v-1}{2}=3 t$ pairwise disjoint almost parallel classes of size $2 t=\frac{v-1}{3}$ (set of pairwise disjoint triples that span $v-1$ elements) and the remaining triples form a partial parallel class of size $t=\frac{v-1}{6}$. We give the number of partial parallel classes and their sizes with respect to $t$, as a Hanani triple system is equivalent to a partial Steiner triple system that admits a color type $(2 t)^{3 t} t^{1}$.

Theorem 3 [6] A HATS(v) exists if and only if $v \equiv 1(\bmod 6)$.

Steiner, Kirkman, and Hanani triple systems have been studied for a long time now, [7, 4, 5, 6] and they are useful for other constructions as we will see in this report.

Another type of configuration that aids in the construction of certain designs is the Group Divisible Design (GDD). A 3-GDD is a collection of 3-elements subsets of a $v$-set $V$, called blocks, which satisfies the following properties;

- each point $v$ appears in $r$ of the $b$ blocks;

- the $v=n l$ elements of $V$ are partitioned into $l$ subsets, which are called groups, each of size $n$;

- no pair of points from the same group occur together in any block;

- any two points not in the same group appear together in $\lambda$ blocks.

We use exponential notation to describe the type of the GDD. So a $G D D\left(g^{u}\right)$ denotes a GDD of type $g^{u}$, which has $u$ groups of size $g$. Staying in the same topic, a Resolvable Group Divisible Design is a GDD whose blocks can be partitioned into parallel classes. An example of an RGDD on 15 points is given in Example 1.1.2.

Example 1.1.2 An $R G D D\left(5^{3}\right)$ with groups, $G_{1}=\{0,1,2,3,4\}, G_{2}=\{5,6,7,8,9\}$, $G_{3}=\{10,11,12,13,14\}$. 


$\begin{array}{lllllllllllllll}0 & 5 & 10 & 0 & 6 & 12 & 0 & 7 & 14 & 0 & 8 & 11 & 0 & 9 & 13 \\ 1 & 6 & 11 & 1 & 7 & 13 & 1 & 8 & 10 & 1 & 9 & 12 & 1 & 5 & 14 \\ 2 & 7 & 12 & 2 & 8 & 14 & 2 & 9 & 11 & 2 & 5 & 13 & 2 & 6 & 10 \\ 3 & 8 & 13 & 3 & 9 & 10 & 3 & 5 & 12 & 3 & 6 & 14 & 3 & 7 & 11 \\ 4 & 9 & 14 & 4 & 5 & 11 & 4 & 6 & 13 & 4 & 7 & 10 & 4 & 8 & 12\end{array}$

A frame parallel class (FPC) for group $G$ is a collection of blocks that partition the points $V \backslash G$, hence it is a partial parallel class that misses every vertex in $G$ but contains every other vertex.

Let $V$ be a finite set, and let $\mathcal{G}$ be a partition of $V$. A 3-frame of type $g_{1}^{u_{1}} g_{2}^{u_{2}} \ldots g_{t}^{u_{t}}$ is a $3-G D D$ of type $g_{1}^{u_{1}} g_{2}^{u_{2}} \ldots g_{t}^{u_{t}}$ together with a partition of its blocks into frame parallel classes. For every group $G \in \mathcal{G}$ there are exactly $\frac{|G|}{2}$ frame parallel classes.

Example 1.1.3 A 3-frame of type $2^{4}$, with groups, $G_{1}=\{1,2\}, G_{2}=\{3,4\}, G_{3}=$ $\{5,6\}, G_{4}=\{7,8\}$. The blocks of this 3-frame are as follows:

\begin{tabular}{|c|c|c|c|}
\hline$F P C 1$ & $F P C 2$ & $F P C 3$ & $F P C 4$ \\
\hline $\begin{array}{lll}3 & 6 & 7\end{array}$ & 168 & $\begin{array}{lll}2 & 3 & 8\end{array}$ & 13 \\
\hline 5 & 25 & 14 & 2 \\
\hline
\end{tabular}

In this report we study the following problem: Does there exist an $S T S(v)$ whose triples can be partitioned into partial parallel classes of size $m$, where $m_{i} \leq\left\lfloor\frac{v}{3}\right\rfloor, m_{i} \mid b$ and $b$ is the number of triples in an $S T S(v)$ ? This question was initially asked by Alex Rosa. Colbourn, Horsley and Wang studied a more general version of this problem in [1]. In this paper they studied the decomposition of partial Steiner Triple Systems, (PSTS) into partial parallel classes of mixed sizes. More specifically they ask, given $v$, $t$ and $m_{1}, m_{2}, \ldots, m_{s}$, with $t=\sum_{i=1}^{s} m_{i}$, does there exist a partial Steiner triple system of order $v$, whose triples can be partitioned into partial parallel classes of sizes $m_{1}, m_{2}, \ldots$, $m_{s}$ ? Elementary necessary conditions are that there exist a partial Steiner triple system of order $v$ having the number of triples equal to the sum of the triples in each partial parallel class. Thus $t=\sum_{i=1}^{s} m_{i}$. Also each partial parallel class can have at most $\left\lfloor\frac{v}{3}\right\rfloor$ triples in it, so $m_{i} \leq\left\lfloor\frac{v}{3}\right\rfloor$ for $1 \leq i \leq s$. These necessary conditions are not sufficient when $v \in\{6,7,9,11,12,13\}$. In the paper they conjecture that these conditions are sufficient for $v \geq 14$. They also show that they are sufficient for $14 \leq v \leq 32$. 
Let $\mu(v)$ denote the number of triples in a partial Steiner triple system of order $v$. This number cannot exceed, $\mu(v)=\left\lfloor\frac{v}{3}\left\lfloor\frac{v-1}{2}\right\rfloor\right\rfloor-\varepsilon$, where $\varepsilon=1$ is $v \equiv 5(\bmod 6)$ and $\varepsilon=0$ otherwise. Then

$$
\mu(v)= \begin{cases}6 t^{2}-2 t & \text { when } v=6 t \\ 6 t^{2}+t & \text { when } v=6 t+1 \\ 6 t^{2}+2 t & \text { when } v=6 t+2 \\ 6 t^{2}+5 t+1 & \text { when } v=6 t+3 \\ 6 t^{2}+6 t+1 & \text { when } v=6 t+4 \\ 6 t^{2}+9 t+2 & \text { when } v=6 t+5\end{cases}
$$

Schönheim [8] showed that a (PSTS) with $\mu(v)$ triples exists for all $v \geq 0$. Such a system is a maximum partial Steiner triple system, $\operatorname{MPT}(v)$.

The basic necessary conditions for a $\operatorname{PSTS}(v)$ to admit a color type $\left(m_{1}, \ldots, m_{c}\right)$ are as follows.

1. For each $1 \leq i \leq c$, there must be a partial parallel class containing $m_{i}$ triples, so then $m_{i} \leq\left\lfloor\frac{v}{3}\right\rfloor$.

2. There must exist a $\operatorname{PSTS}(v)$ having $\sum_{i=1}^{c} m_{i}$ triples, so $\sum_{i=1}^{c} m_{i} \leq \mu(v)$.

The interest of the authors in [1] is to determine the possible color types of block colorings, (the possible decomposition of the blocks) of a PSTS $(v)$. They conjecture the following.

Conjecture 4 Let $v \geq 14$. Let $\left(m_{1}, \ldots, m_{c}\right)$ satisfy $\sum_{i=1}^{c} m_{i} \leq \mu(v)$ and $m_{i} \leq\left\lfloor\frac{v}{3}\right\rfloor$ for $1 \leq i \leq c$. Then there exists a partial Steiner triple system that admits a block coloring of color type $\left(m_{1}, \ldots, m_{c}\right)$.

In the paper they divide the coloring of the triples in two cases. They deal first with orders up to 13 . They next consider the orders $14 \leq v \leq 32$, where they prove their conjecture all such $v$.

We will call a decomposition in which each color class has the same size, uniform. Thus in the context of their paper a Kirkman triple system $\operatorname{KTS}(6 t+3)$, is equivalent to a PSTS $(6 t+3)$ that admits a uniform color type $(2 t+1)^{3 t+1}$.

A partial Steiner triple system whose triples can be partitioned into $s$ partial parallel classes, each of size $m$, is a signal set, denoted $S S(v, s, m)$. So a signal set $S S(v, s, m)$ 
is a $\operatorname{PSTS}(v)$ that admits a color type $m^{s}$. When $s=\left\lfloor\frac{\mu(v)}{m}\right\rfloor$ the $S S(v, s, m)$ is a Kirkman signal set, $K S S(v, m)$. Many of the uniform decompositions are covered by the next result.

Theorem 5 [9] A KSS $\left(v,\left\lfloor\frac{v}{3}\right\rfloor\right)$ exists for each positive integer $v$ such that $v \notin\{6,7,12\}$.

A major construction technique for many of the non-uniform decompositions relies on the idea of dismantling closely related partial parallel classes that have been constructed previously on $v$ points. We examine this technique more carefully in Chapter 2 of this report. Colbourn et. al. in [1], study the problem partitioning partial Steiner triple systems into partial parallel classes of mixed sizes. In this report, we take into consideration only Steiner triple systems, $|\{B \in \mathcal{B}:\{x, y\} \subset B\}|=1$. Equivalent to the signal sets, we want to partition the $b$ blocks of an $S T S(v)$ into $s$ partial parallel classes, all of size $m$, where, $s m=b=\frac{v(v-1)}{6}$ and $m \leq \frac{v}{3}$. Thus we focus on the uniform case of the problem.

\subsection{Preliminary Results}

We denote by a $S T S_{m}(v)$ a $S T S(v)$ whose blocks can be partitioned into partial parallel classes of size $m$. The next three results are straightforward, and can be found in [1].

Lemma 6 [1] If $v \equiv 3(\bmod 6)$, then there exists a $S T S_{\frac{v}{3}}(v)$.

Proof If $v \equiv 3(\bmod 6)$ then the $S T S(v)$ is a $K T S(v)$, and a Kirkman triple system is just a resolvable Steiner triple system. This means that we can partition its blocks into parallel classes of size $m=\frac{v}{3}$, and in total we would have $\frac{v-1}{2}$ parallel classes.

Lemma 7 [1] Suppose $m_{1} \mid m$ and there exists an $S T S_{m}(v)$, then there exists an $S T S_{m_{1}}(v)$.

Proof The proof is obvious. We divide each partial parallel class of size $m$ into partial parallel classes of size $m_{1}$.

Recall a Hanani triple system, $\operatorname{HATS}(6 t+1)$ is equivalent to an $S T S(6 t+1)$ that admits a color type $(2 t)^{3 t} t^{1}$. It was shown in [6] that a Hanani triple system exists if and only if $v \equiv 1(\bmod 6)$ and $v \notin\{7,13\}$. So we will have $3 t$ partial parallel classes with $2 t=\frac{v-1}{3}$ blocks in them and a partial parallel class with $t=\frac{v-1}{6}$ blocks. We partition each one of the $3 t$ partial parallel classes of size $2 t$ in two. We then have $6 t+1=6 \cdot \frac{v-1}{6}+1=v$ partial parallel classes of size $t=\frac{v-1}{6}$. Hence the following result. 
Lemma 8 [1] If $v=6 t+1$, then there exists a $S T S_{t}(v)$.

An $l-P P C$ denotes a partial parallel class with $l$ triples in it.

The following two theorems give results on decomposing an $S T S(v)$ into partial parallel classes.

Theorem 9 [3] Let $S=(V, \mathcal{B})$ be an $S T S(v)$ with $v>9 l$. Then there exists a decomposition of $\mathcal{B}$ into $l-P P C^{\prime} s$.

Theorem 10 [3] An STS $(v)$ can be decomposed into 2-PPC's if and only if $2 \mid b$ and $v \neq 9$.

From the previous theorem we know we are able to decompose an $S T S(v)$ into partial parallel classes of size 2. In Chapter2, we develop a method of decomposing the blocks of an $S T S(v)$ into $2-P P C^{\prime} s$, and we then extend this method to $4-P P C^{\prime} s$. We also give a few special decompositions in which the techniques used could possibly be generalized.

Chapter 3 summarizes our results and gives a table of what is known on the problem for $v \leq 43$, and discusses some options for future research. 


\section{Chapter 2}

\section{New results}

In this chapter we study one of the main techniques used in [1] to decompose the blocks of an $S T S(v)$ into $l-P P C^{\prime} s$. We give the result, and illustrate the idea with the example $v=25, m=5$. Although the technique is used to obtain many of the non-uniform decompositions, it will also be useful for the uniform case.

Theorem 9 says we can always decompose an $S T S(v)$, when $b=\frac{v(v-1)}{2}$ into partial parallel classes of size 2 except for the cases when $v=9$. We develop a different technique to do so, that could possibly be generalized for partial parallel classes of larger size. We extend the idea behind this technique, to partial parallel classes of size 4. We also give the construction of a $5-P P C$ decomposition for $v=21$ and $v=25$.

\subsection{Dismantling Partial Parallel Classes}

The following method was used in [1] to construct partial parallel classes decomposition of new sizes from already known decompositions. The idea behind this method is given in the following lemmas. The first one dismantles two partial parallel classes to form three partial parallel classes.

Lemma 11 [1] Let $n_{1}$ and $n_{2}$ be positive integers such that $n_{1} \leq n_{2}$, let $V_{1}$ and $V_{2}$ be disjoint sets such that $\left|V_{1}\right|=n_{1}$ and $\left|V_{2}\right|=n_{2}$, and let $G$ be a bipartite graph with bipartition $\left(V_{1}, V_{2}\right)$ such that $G$ has maximum degree at most 3 and either $G$ is connected or $n_{2} \leq 2 n_{1}$. Let $x_{1}$ and $x_{2}$ be integers such that either $x_{1} \leq n_{1}$ and $2 x_{1}+x_{2}<n_{2}$ or $x_{1}=x_{2}=\frac{n_{1}}{3}=\frac{n_{2}}{3}$. Then unless $G$ is $K_{3,3}$ and $x_{1}=x_{2}=1$, there are sets $X_{1} \subseteq V_{1}$ and $X_{2} \subseteq V_{2}$ such that $\left|X_{1}\right|=x_{1},\left|X_{2}\right|=x_{2}$ and no edge of $G$ has one end in $X_{1}$ and one end in $X_{2}$.

Lemma 12 [1] Suppose a color type $T^{\prime}$ can be obtained from a color type $T$ by iteratively applying the following operations: 
(i) Take an entry $y$ in the sequence and replace it with an entry a such that $a \leq y$.

(ii) Take an entry $y$ in the sequence and replace it with two entries $a$ and $b$ such that $a+b=y$.

(iii) Take two entries $y$ and $z$ such that $y \leq z \leq 2 y$ and replace them with three entries $a, b$, and c such that $a+b+c=y+z, a \leq y, b \leq z,(a, b, c) \neq(2,2,2)$ and either $2 y<2 a+b$ or $a=b=c=\frac{2 y}{3}=\frac{2 z}{3}$.

Then, if $(V, \mathcal{B})$ is a PSTS that admits color type $T$, there is a PSTS $\left(V, \mathcal{B}^{\prime}\right)$ with $\mathcal{B}^{\prime} \subseteq \mathcal{B}$ that admits a color type $T^{\prime \prime}$.

Lemmma 12 may be used to obtain uniform decompositions when certain mixed decompostions are known. The following example illustrates the lemma by obtaining a decomposition of an $S T S(25)$ into $5-P P C^{\prime} s$ from the known decomposition with color type $8^{6} 7^{6} \quad 5^{2}$.

Example 2.1.1 A decomposition of an $S T S(25)$ into $5-P P C^{\prime}$ s.

From the results of the paper we studied, [1], there is an STS(25) that has a color type $T=8^{6} 7^{6} 5^{2}$. We want to get a color type $T^{\prime}=5^{20}$, in other words we want 20 partial parallel classes of size 5. In order to get the second color type or the $205-$ P PCs of the STS(25) we apply Lemma 12

First we choose $y=7$ and $z=8$. Then we let $a=b=c=5$. We check if the conditions stated in the lemma hold.

1. $7 \leq 8 \leq 2 \cdot 7 \Rightarrow y \leq z \leq 2 y$

2. $5+5+5=7+8 \Rightarrow a+b+c=y+z$

3. $5 \leq 7 \Rightarrow a \leq y$

4. $5 \leq 8 \Rightarrow b \leq z$

5. $2 \cdot 7 \leq 2 \cdot 5+5 \Rightarrow 2 y \leq 2 a+b$

In this way, each time we have a partial parallel class of size 8 and a partial parallel class of size 7 we'll get 3 partial parallel classes of size 5. From the color type $T=$ $8^{6} \quad 7^{6} 5^{2}$ that we have, we get 18 partial parallel classes of size 5 from the 6 partial parallel classes of size 8 and the 6 classes of size 7. There were already 2 partial parallel classes of size 5. Thus in total we have 20 partial parallel classes of size 5. This STS(25) with color type $T^{\prime}=5^{20}$ has been constructed, i.e. there exists an $\operatorname{ST~}_{5}(25)$. 
Example 2.1.2 A decomposition of an $S T S(13)$ into $2-P P C^{\prime} s$.

In [1] we can find that there exists an STS(13) that has a color type $T=4^{4} 2^{5}$. We want to get a color type $T^{\prime}=2^{13}$, or 13 partial parallel classes of size 2 . By partitioning the 4 partial parallel classes of size 4 of the color type $T=4^{4} 2^{5}$ in two, we would get 8 partial parallel classes of size 2. We already have 5 other partial parallel classes of size 2. Thus we would have in total 13 partial parallel classes of size 2, or $132-P P C s$.

\subsection{New Results}

The main results of this chapter use 3-frames. Stinson solved the existence problem for 3-frames in 1987.

Lemma 13 [4] There exists a 3-frame of type $g^{u}$ if and only if $g$ is even, $u \geq 4$, and $g(u-1) \equiv 0(\bmod 3)$.

\subsubsection{Partial Parallel classes of size $m=2$}

Lemma 14 If there exists a 3-frame of type $2^{u}$ and $u \geq 7$, then there exists an $S T S_{2}(2 u+1)$, whenever $b=\frac{(2 u+1)(2 u)}{6}=\frac{u(2 u+1)}{3}$ is even.

Proof Let $v=2 u+1$ and $G_{1}, G_{2}, \ldots, G_{u}$, be the groups of a 3-frame of type $2^{u}$. The blocks of the 3-frame along with the blocks formed as $G_{i} \cup\{\infty\}$, for $i=1,2, \ldots, u$ forms a $S T S(2 u+1)$. To see this, we need to show that each pair is in exactly one block. In a 3-frame we have that each pair from distinct groups is in exactly one block, and no pairs from the same group are in the same block. From the blocks created as $G_{i} \cup\{\infty\}$, we have that the pairs in one group will be in exactly one block as well. All the pairs containing $\{\infty\}$ will be covered exactly once also. So every pair of the $S T S(2 u+1)$ is in exactly one block. The number of blocks in a $S T S(v)$ is $\frac{v(v-1)}{6}$, so we will have $\frac{v(v-1)}{12}$ partial parallel classes.

Now we describe the partial parallel classes. Because a 3-frame of type $2^{u}$ exists, we must have $u \equiv 1(\bmod 3)$. But when $u \equiv 1(\bmod 6), 2 \nmid b$, hence $u \equiv 4(\bmod 6)$, and in particular $u$ is even. We partition the groups into pairs:

$$
\left\{G_{1}, G_{2}\right\},\left\{G_{3}, G_{4}\right\}, \ldots,\left\{G_{u-1}, G_{u}\right\} .
$$

For each pair of groups $G_{i}$ and $G_{j}$, consider the frame parallel classes $F_{i}$ and $F_{j}$. In $F_{i}$ there is at least one block $B$ which is disjoint with $G_{j}$. In each frame parallel class 
there are $\frac{v-3}{3}$ blocks. There are two blocks in $F_{i}$ that intersect $G_{j}$. Thus we are left with $\frac{v-9}{3}$ blocks that do not intersect $G_{j}$. Let $B$ be one of these blocks.

Remove $B$ from $F_{i}$ and add it to $F_{j}$. The number of blocks remaining in $F_{i}$ now is:

$$
\frac{2 u-2}{3}-1=\frac{2 u-5}{3}=\frac{2 \frac{v-1}{2}-5}{3}=\frac{v-6}{3}=\frac{v}{3}-2
$$

Since $v \equiv 3(\bmod 6)$ this number is odd. Partition $G_{i} \cup\{\infty\}$ along with the remaining blocks of $F_{i}$ into partial parallel classes of size 2 . The number of blocks in $F_{j}$ is:

$$
\frac{2 u-2}{3}=\frac{2 \frac{v-1}{2}-2}{3}=\frac{v-3}{3}
$$

which is even. Because $G_{j} \cap B=\emptyset$, we may take $G_{j} \cup\{\infty\}$ and $B$ to be a partial parallel class of size 2. Partition the rest of $F_{j}$ into $2-P P C^{\prime} s$.

This accounts for: $\frac{\frac{v}{3}-2+1}{2}=\frac{v-3}{6}$ partial parallel classes in $F_{i}$ and $\left(\frac{v-3}{3}+2\right) \cdot \frac{1}{2}=\frac{v+3}{6}$ partial parallel classes in $F_{j}$. So in $F_{i}$ and $F_{j}$ together we have:

$$
\frac{v-3}{6}+\frac{v+3}{6}=\frac{2 v}{6}=\frac{v}{3}
$$

partial parallel classes.

We have $\frac{u}{2}$ pairs of groups, and each of them gives us $\frac{v}{3}$ partial parallel classes. So in total we get:

$$
\frac{v}{3} \cdot \frac{u}{2}=\frac{v}{3} \cdot \frac{v-1}{4}=\frac{v(v-1)}{12}
$$

partial parallel classes, the number we were looking for.

Theorem 15 If $2 \mid b$, then there exists an $S T S(v)$ whose blocks can be partitioned into $\frac{b}{2} 2-P P C s$.

Proof If $v \equiv 1(\bmod 6)$, then $b=\frac{v(v-1)}{3 \cdot 2} \equiv 0(\bmod 2)$ when $\frac{v-1}{6}$ is even. Let $v=6 t+1$, then $b \equiv 0(\bmod 2)$ whenever $t$ is even. By Theorem 3 , there exists a $H A T S(6 t+1)$ for all such $t$, except for $t \in\{1,2\}$. This is equivalent to an $S T S(6 t+1)$ with color type $(2 t)^{3 t} t^{1}$. Because $t$ is even, we can divide each partial parallel class into $2-P P C^{\prime} s$. If $t=1, b$ is not even. If $t=2$, then an $S T S_{2}(13)$ exists by Lemma 12 
If $v \equiv 3(\bmod 6)$ and $v \geq 15$, then $\frac{v-1}{2} \equiv 1,4(\bmod 6)$, and $\frac{v}{3} \equiv 1,3,5(\bmod 6)$. Therefore, $b=\frac{v(v-1)}{3 \cdot 2} \equiv 0(\bmod 2)$ only when $\frac{v-1}{2} \equiv 4(\bmod 6)$. So let $u=\frac{v-1}{2} \equiv 4$ $(\bmod 6)$. Then $u-1 \equiv 0(\bmod 3)$, and by Lemma 13 , there exists a 3 -frame of type $2^{u}$. Now we apply Lemma 14 to construct the $2-P C^{\prime} s$.

If $v=7$ and $v=9$, then by Theorem 10 neither the $S T S(7)$ nor the $S T S(9)$ can be partitioned into $2-P P C^{\prime} s$.

\subsubsection{Partial parallel classes of size $m=4$}

Lemma 16 If there exists a 3-frame of type $2^{u}$, and $u \geq 16$, then there exists an $S T S_{4}(2 u+1)$, whenever $4 \mid b$.

Proof Let $v=2 u+1$ and $G_{1}, G_{2}, \ldots, G_{u}$, be the groups of a 3-frame of type $2^{u}$. The blocks of the 3-frame along with the blocks formed as $G_{i} \cup\{\infty\}$, for $i=1,2, \ldots, u$ forms a $S T S(2 u+1)$. The proof for this is identical to the proof in Lemma 14. Again this $S T S(2 u+1)$ has $\frac{v(v-1)}{6}$ blocks, so we will require $\frac{v(v-1)}{24}$ partial parallel classes. Because of the existence of a 3 -frame of type $2^{u}$, we must have that $u \equiv 1(\bmod 3)$. This means that $u \equiv 1,4(\bmod 6)$. But when $u \equiv 1(\bmod 6), b \neq \equiv 0(\bmod 2)$. So $u \equiv 4(\bmod 6)$, and in particular $u$ is even. However $u$ being even is not enough, since we want that $4 \mid b$. If $u \equiv 4(\bmod 6)$, then $u \equiv 0,2(\bmod 4) \cdot u \equiv 4(\bmod 6)$, $u \equiv 0(\bmod 4)$ by the Chinese Remainder Theorem, $u \equiv 4(\bmod 12)$. We partition the groups into sets of 4 :

$$
\left\{G_{1}, G_{2}, G_{3}, G_{4}\right\},\left\{G_{5}, G_{6}, G_{7}, G_{8}\right\}, \ldots,\left\{G_{u-3}, G_{u-2}, G_{u-1}, G_{u}\right\}
$$

For each set of 4 groups $G_{i}, G_{j}, G_{k}, G_{s}$ for instance, consider the corresponding frame parallel classes $F_{i}, F_{j}, F_{k}$, and $F_{s}$. In each frame parallel class there are $\frac{v-3}{3}$ blocks. There are two blocks in $F_{i}$ that intersect $G_{j}$. Thus we are left with $\frac{v-9}{3} \geq 8$ blocks that do not intersect with $G_{j}$. Let $B_{j}$ be one of these blocks. Remove $B_{j}$ from $F_{i}$ and add it to $F_{j}$. There are 3 blocks in $F_{j}$ that intersect $B_{j}$. Pick two blocks $B_{j_{1}}$ and $B_{j_{2}}$ that are not any of these.

So we would get this partial parallel class of size $4 ;\left\{G_{j} \cup\{\infty\}, B_{j}, B_{j_{1}}, B_{j_{2}}\right\}$. The blocks $B_{j_{1}}$, and $B_{j_{2}}$ are in frame $F_{j}$. We take two more blocks from $F_{i}$ and add them to $F_{k}$ and $F_{s}$ respectively. Following the same reasoning as above, we can find these two more blocks that do not intersect with $G_{k}$ and $G_{s}$. So we also remove $B_{k}$ and $B_{s}$ from $F_{i}$ and add them to $F_{k}$ and $F_{s}$ respectively. In this way we would form two more partial parallel classes of size 4 . After removing these 3 blocks from $F_{i}$ the number of remaining blocks is: 


$$
\frac{2 u-2}{3}-3=\frac{2 \frac{v-1}{2}-2}{3}-3=\frac{v-3-9}{3}=\frac{v}{3}-4
$$

Because $v \equiv 3(\bmod 6)$, we have that $\frac{v}{3}-4$ is odd.

Partition $G_{i} \cup\{\infty\}$ along with the remaining blocks of $F_{i}$ into partial parallel classes of order 4 . The number of partial parallel classes in $F_{i}$ is:

$$
\frac{\frac{v}{3}-4+1}{4}=\frac{\frac{v}{3}-3}{4}=\frac{v-9}{12}
$$

The number of blocks in $F_{j}, F_{k}$, and $F_{s}$ after adding up the blocks we took from frame $F_{i}$ will be

$$
\frac{2 u-2}{3}+1=\frac{2 \frac{v-1}{2}-2}{3}+1=\frac{v-3+3}{3}=\frac{v}{3}
$$

We partition $G_{j} \cup\{\infty\}, G_{k} \cup\{\infty\}$, and $G_{s} \cup\{\infty\}$ along with the rest of the blocks in their respective frames, and we get

$$
\left(\frac{v}{3}+1\right) \cdot \frac{1}{4}=\frac{v+3}{12}
$$

partial parallel classes. In these 4 frames together, $F_{i}, F_{j}, F_{k}$, and $F_{s}$ we have in total

$$
\frac{v-9}{12}+3 \cdot \frac{v+3}{12}=\frac{v-9+3 v+9}{12}=\frac{4 v}{12}=\frac{v}{3}
$$

partial parallel classes. We have $\frac{u}{4}$ sets of groups, each of them gives us $\frac{v}{3}$ partial parallel classes. Thus the total number of partial parallel classes is

$$
\frac{v}{3} \cdot \frac{u}{4}=\frac{v}{3} \cdot \frac{v-1}{8}=\frac{v(v-1)}{24}
$$

partial parallel classes, the number we were looking for.

Theorem 17 If $4 \mid b$, then there exists an $S T S(v)$ whose blocks can be partitioned into $\frac{b}{4} 4-P P C s$.

Proof If $v \equiv 1(\bmod 6)$, then we should have that $\left(\frac{v-1}{6}\right) \equiv 0(\bmod 4)$, in order that $4 \mid b$. Let $v=6 t+1$, then we need $t \equiv 0(\bmod 4)$. From Theorem 3 there exists a $H A T S(6 t+1)$ for all such $t$. This $\operatorname{HATS}(6 t+1)$ is equivalent to an $S T S(6 t+1)$ 
that admits a color type $(2 t)^{3 t} t^{1}$. Now since $t \equiv 0(\bmod 4)$ we can divide each of these partial parallel classes into $4-P P C^{\prime} s$.

If $v \equiv 3(\bmod 6)$ then we have that $\frac{v-1}{2} \equiv 1,4(\bmod 6)$ and $\frac{v}{3} \equiv 1,3,5(\bmod 6)$ and so $\frac{v}{3}$ is odd. Therefore because $4 \mid b$ when $\frac{v-1}{2} \equiv 0(\bmod 4)$ and thus $u=\frac{v-1}{2} \equiv 4$ $(\bmod 6)$. Consequently $u-1 \equiv 0(\bmod 3)$, and so by Lemma 13 , there exists a 3 frame of type $2^{u}$. Now apply Lemma 16 to construct the $4-P P C s$. 


\subsection{Special Cases}

Theorems 15 and 17 cover the decomposition of many cases. However there are cases when we cannot use these results. We discuss some of those cases now. The case of $v=21$ and $m=5$ has been covered in [1], but the case of $v=33$ and $m=8$ has not. We give our own construction for each of these.

\subsubsection{When $v=21$ and $m=5$}

Lemma 18 There exists an $S T S_{5}(21)$.

In this case we have an $S T S(21)$, which is actually a $K T S(21)$. We will have to decompose the 70 blocks into 14 partial parallel classes of size 5 . We partition the points into 3 groups; $G_{0}=\left\{0_{0}, 1_{0}, 2_{0}, 3_{0}, 4_{0}, 5_{0}, 6_{0}\right\}, G_{1}=\left\{0_{1}, 1_{1}, 2_{1}, 3_{1}, 4_{1}, 5_{1}, 6_{1}\right\}$, $G_{2}=\left\{0_{2}, 1_{2}, 2_{2}, 3_{2}, 4_{2}, 5_{2}, 6_{2}\right\}$. Since we are working on 21 points, $21=7 \cdot 3$, we can put an $S T S(7)$ in each of the groups. We work on each group cyclically $(\bmod 7)$.

Because we are working $(\bmod 7)$ in each of the 3 groups, then we will have these differences, $\{1,2,3\}$ between the points in each of the groups. We will also consider the differences between the points of one group with the points of 2 other groups. We will call these type of differences, "cross differences".

The blocks of the partial parallel classes will be formed in the way that the differences between the points in each block cover all the differences among the 7 points in each group, i.e. $\{1,2,3\}$. The rest of the blocks will be formed such that the points of each block cover the cross differences between the groups $G_{0}, G_{1}$, and $G_{2}$, i.e. $\{0,1,2,3,4,5,6\}$. Below we give all the 14 partial parallel classes of size 5 .

\begin{tabular}{|c|c|c|c|}
\hline$P P C 1$ & PPC2 & PPC3 & PPC4 \\
\hline $\begin{array}{lll}0_{0} & 1_{0} & 3_{0}\end{array}$ & $1_{0} \quad 2_{0} \quad 4_{0}$ & $\begin{array}{lll}2_{0} & 3_{0} & 5_{0}\end{array}$ & $\begin{array}{lll}3_{0} & 4_{0} & 6_{0}\end{array}$ \\
\hline $0_{1} \quad 1_{1}$ & $1_{1} \quad 2_{1} \quad 4_{1}$ & $\begin{array}{lll}2 & 3_{1} & 5_{1}\end{array}$ & $3_{1}$ \\
\hline $1_{2}$ & $2_{2}$ & $3_{2}$ & $3_{2}$ \\
\hline $2_{1}$ & $3_{0}$ & $4_{1}$ & $5_{0}$ \\
\hline $\begin{array}{lll}4_{0} & 5_{1} & 6_{2}\end{array}$ & $\begin{array}{lll}5_{0} & 6_{1} & 0_{2}\end{array}$ & $\begin{array}{lll}6_{0} & 0_{1} & 1_{2}\end{array}$ & $1_{1} \quad 2_{2}$ \\
\hline
\end{tabular}




\begin{tabular}{|c|c|c|c|}
\hline$P P C 5$ & $P P C 6$ & PPC7 & PPC8 \\
\hline $0_{0} \quad 4_{0} \quad 5_{0}$ & $1_{0} \quad 5_{0} \quad 6_{0}$ & $\begin{array}{lll}0_{0} & 2_{0} & 6_{0}\end{array}$ & $\begin{array}{lll}0_{0} & 2_{1} & 4_{2}\end{array}$ \\
\hline $0_{1} \quad 4_{1} \quad 5_{1}$ & $1_{1} \quad 5_{1} \quad 6_{1}$ & $\begin{array}{lll}0_{1} & 2_{1} & 6_{1}\end{array}$ & $1_{0} \quad 3_{1}$ \\
\hline $0_{2} \quad 4_{2} \quad 5_{2}$ & $1_{2} \quad 5_{2} \quad 6_{2}$ & $\begin{array}{lll}0_{2} & 2_{2} & 6_{2}\end{array}$ & $2_{0}$ \\
\hline $\begin{array}{lll}6_{0} & 6_{1} & 6_{2}\end{array}$ & $\begin{array}{lll}0_{0} & 0_{1} & 0_{2}\end{array}$ & $\begin{array}{lll}1_{0} & 1_{1} & 1_{2}\end{array}$ & $3_{0} \quad 5_{1}$ \\
\hline $1_{0} \quad 2_{1} \quad 3_{2}$ & $2_{0} \quad 3_{1} \quad 4_{2}$ & $3_{0} \quad 4_{1} \quad 5_{2}$ & $4_{0}$ \\
\hline PPC9 & $P P C 10$ & $P P C 11$ & PPC12 \\
\hline $\begin{array}{lll}0_{0} & 3_{1} & 6_{2}\end{array}$ & $\begin{array}{lll}0_{0} & 4_{1} & 1_{2}\end{array}$ & $1_{0} \quad 5_{1} \quad 2_{2}$ & $\begin{array}{lll}0_{0} & 5_{1} & 3_{2}\end{array}$ \\
\hline $\begin{array}{lll}1_{0} & 4_{1} & 0_{2}\end{array}$ & $\begin{array}{lll}3_{0} & 6_{1} & 2_{2}\end{array}$ & $\begin{array}{lll}2_{0} & 6_{1} & 3_{2}\end{array}$ & $1_{0} \quad 6_{1}$ \\
\hline $\begin{array}{lll}2_{0} & 5_{1} & 1_{2}\end{array}$ & $4_{0} \quad 0_{1}$ & $\begin{array}{lll}3_{0} & 0_{1} & 4_{2}\end{array}$ & $2_{0}$ \\
\hline $\begin{array}{ll}5_{0} & 0_{1}\end{array}$ & $5_{0} \quad 1_{1}$ & $\begin{array}{lll}4_{0} & 1_{1} & 5_{2}\end{array}$ & $3_{0}$ \\
\hline $\begin{array}{lll}6_{0} & 1_{1} & 3_{2}\end{array}$ & $6_{0} \quad 2_{1}$ & $\begin{array}{lll}5_{0} & 2_{1} & 6_{2}\end{array}$ & $6_{0}$ \\
\hline
\end{tabular}

\begin{tabular}{ccccccc}
\multicolumn{3}{c}{$P P C 13$} & \multicolumn{4}{c}{$P P C 14$} \\
$0_{0}$ & $6_{1}$ & $5_{2}$ & $2_{0}$ & $1_{1}$ & $0_{2}$ \\
$1_{0}$ & $0_{1}$ & $6_{2}$ & $3_{0}$ & $2_{1}$ & $1_{2}$ \\
$4_{0}$ & $2_{1}$ & $0_{2}$ & $4_{0}$ & $3_{1}$ & $2_{2}$ \\
$5_{0}$ & $3_{1}$ & $1_{2}$ & $5_{0}$ & $4_{1}$ & $3_{2}$ \\
$6_{0}$ & $4_{1}$ & $2_{2}$ & $6_{0}$ & $5_{1}$ & $4_{2}$
\end{tabular}

Figures 2.1 - 2.5 illustrate the 14 partial parallel classes of size 5 of the $S T S(21)$. The blocks $\left\{0_{0}, 1_{0}, 3_{0}\right\},\left\{0_{1}, 1_{1}, 3_{1}\right\}$, and $\left\{0_{2}, 1_{2}, 3_{2}\right\}$ colored in red in Figure 2.1 cover

the differences 1,2 , and 3 in group $G_{0}, G_{1}$, and $G_{2}$. After developing these blocks $(\bmod 7)$ all edges with differences 1,2 , and 3 have been covered.

The block $\left\{2_{0}, 2_{1}, 2_{2}\right\}$ colored in green in Figure 2.1 covers the cross differences $(0,0$, 0 ) between the group $G_{0}, G_{1}$, and $G_{2}$. When we develop $(\bmod 7)$ this base block we will have covered all edges with cross difference 0 .

This block $\left\{4_{0}, 5_{1}, 6_{2}\right\}$, colored in blue in Figure 2.1 covers edges with cross differences $(1,1,5)$ between the groups $G_{0}$ and $G_{1}, G_{1}$ and $G_{2}, G_{2}$ and $G_{0}$ respectively. Developing this base block $(\bmod 7)$ we cover all the $(1,1,5)$ cross differences between the three groups.

On the second partial parallel class in Figure 2.3 we cover the cross differences (2, 2,3 ) between the groups $G_{0}$ and $G_{1}, G_{1}$ and $G_{2}, G_{2}$ and $G_{0}$ respectively. The block 
$\left\{0_{0}, 2_{1}, 4_{2}\right\}$ developed ( $\left.\bmod 7\right)$ will give us all such differences. Two more blocks that cover these cross differences are given in red, on the third partial parallel class in Figure 2.3.

Continuing on Figure 2.3, the rest of the blocks on the third partial parallel class, given in blue cover the cross differences $(3,3,1)$ between the groups $G_{0}$ and $G_{1}, G_{1}$ and $G_{2}$, $G_{2}$ and $G_{0}$. We have four more such blocks on the first partial parallel class of Figure 2.4. colored in blue. By developing $(\bmod 7)$ the block $\left\{0_{0}, 3_{1}, 6_{2}\right\}$ we will get all such differences between the three groups.

The next cross differences being covered by the green blocks in Figure 2.4 are $(4,4,6)$. Considering the block $\left\{0_{0}, 4_{1}, 1_{2}\right\}$ (mod 7$)$, we will get all these differences between the groups $G_{0}$ and $G_{1}, G_{1}$ and $G_{2}, G_{2}$ and $G_{0}$.

The third partial parallel class in Figure 2.4 has four blocks, that are colored in purple, which cover the cross differences $(5,5,4)$ between the groups $G_{0}$ and $G_{1}, G_{1}$ and $G_{2}$, $G_{2}$ and $G_{0}$. We have three more such blocks on the first partial parallel class of Figure 2.5. The base block of these differences is $\left\{0_{0}, 5_{1}, 3_{2}\right\}$.

The last cross differences that are being covered in Figure 2.5 between the groups $G_{0}$ and $G_{1}, G_{1}$ and $G_{2}, G_{2}$ and $G_{0}$, are the differences $(6,6,2)$. All these blocks are give in blue. The base block of these differences is $\left\{0_{0}, 6_{1}, 5_{2}\right\}$.

As we can see all the differences within the groups and between the groups, the cross differences, are covered the same number of times. Having all the differences means that we have all the blocks of the $S T S(21)$, and all of them are being partitioned into partial parallel classes of size 5 . 

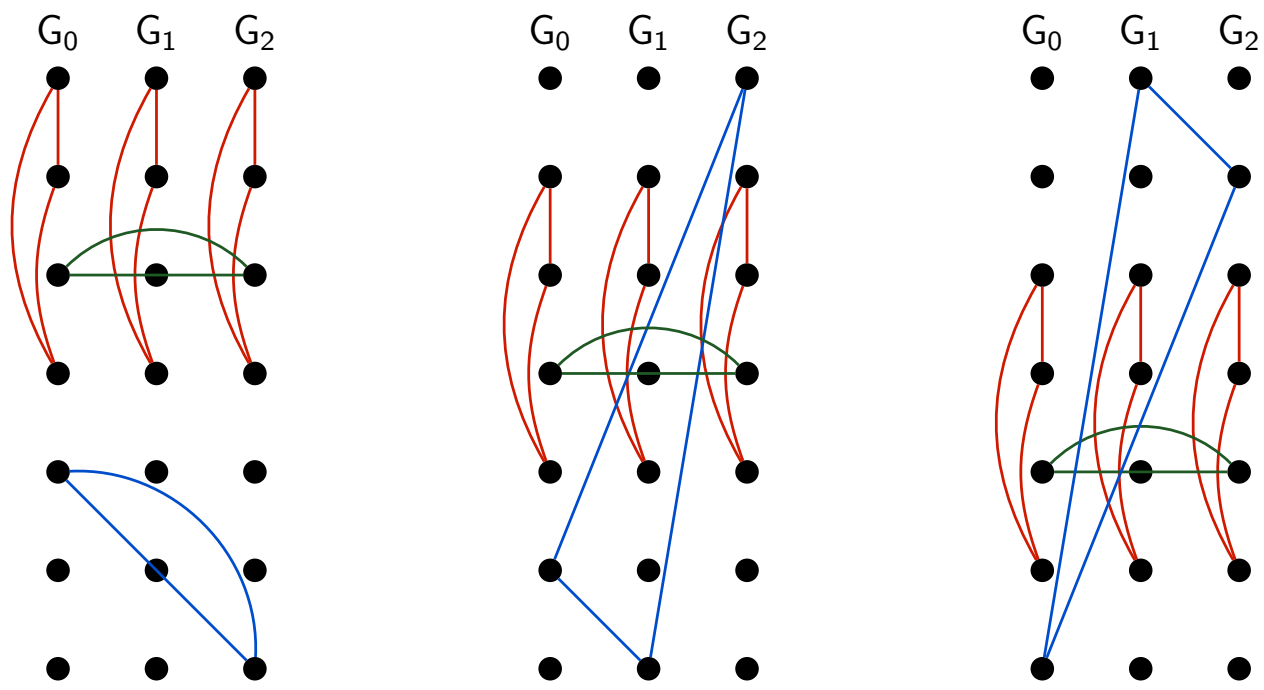

Figure 2.1: Partial parallel classes of size 5
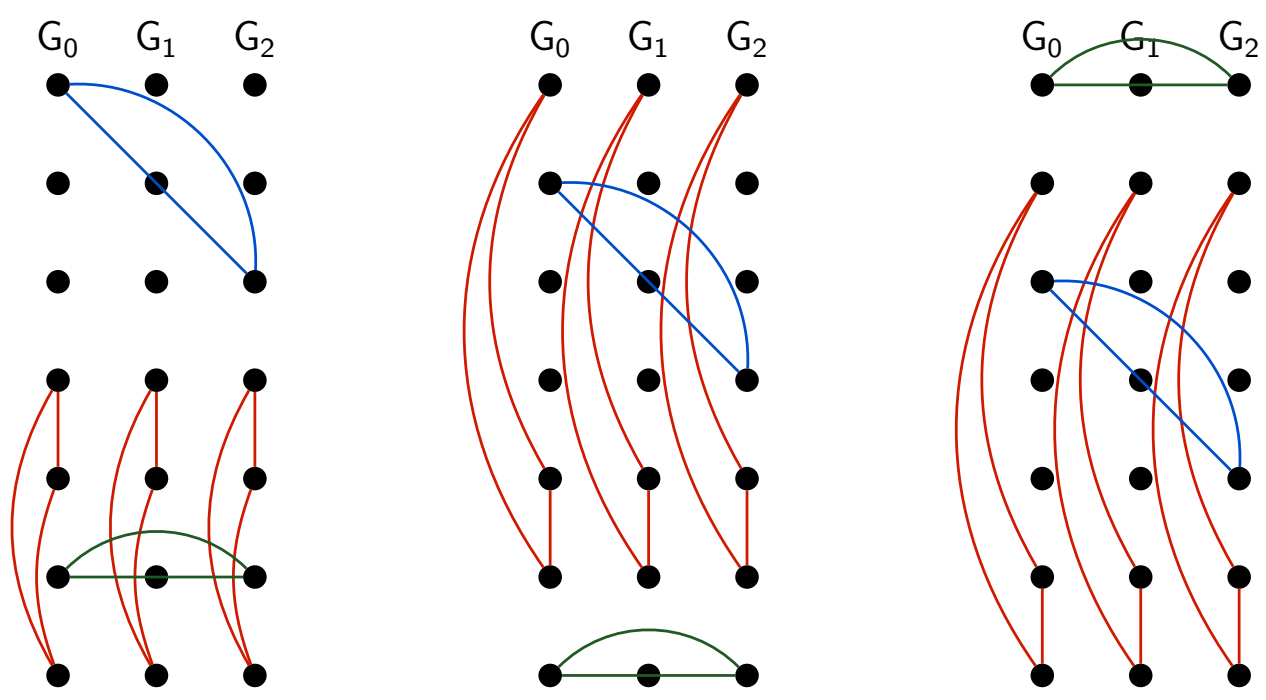

Figure 2.2: Partial parallel classes of size 5 

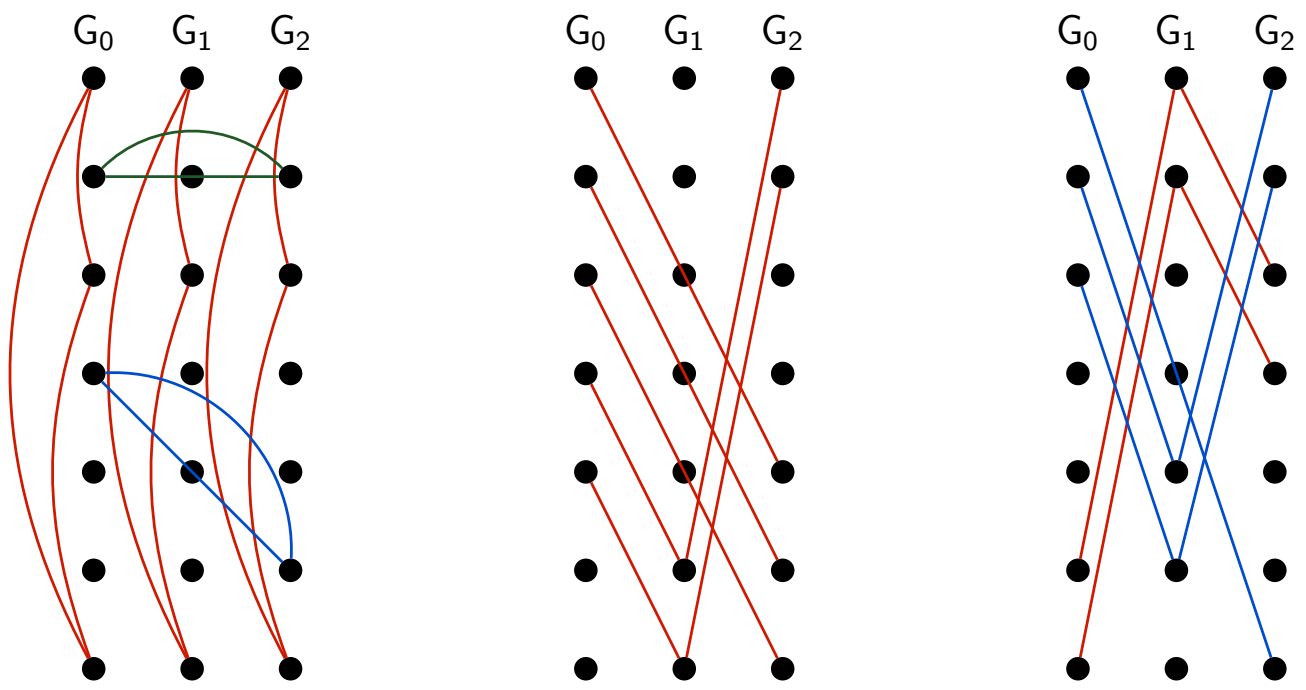

Figure 2.3: Partial parallel classes of size 5
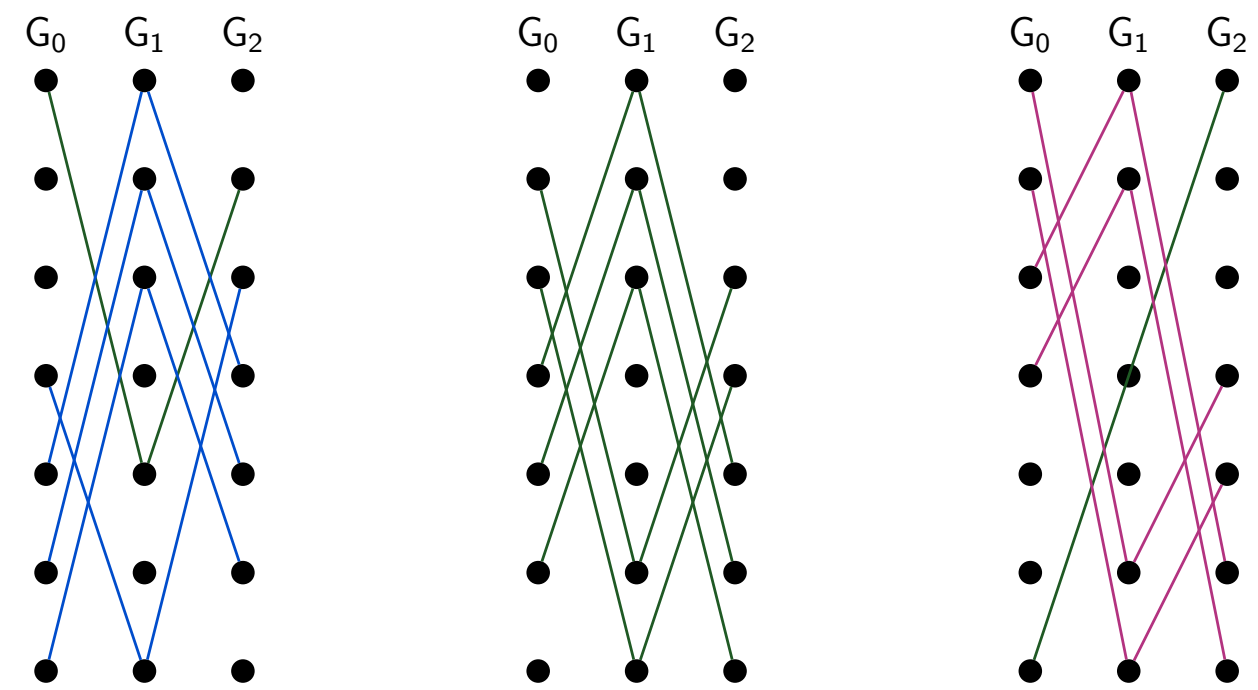

Figure 2.4: Partial parallel classes of size 5

\subsubsection{When $v=33$ and $m=8$}

In this construction, we use a resolvable GDD. Rees solved the existence problem for RGDD's with block size 3. 

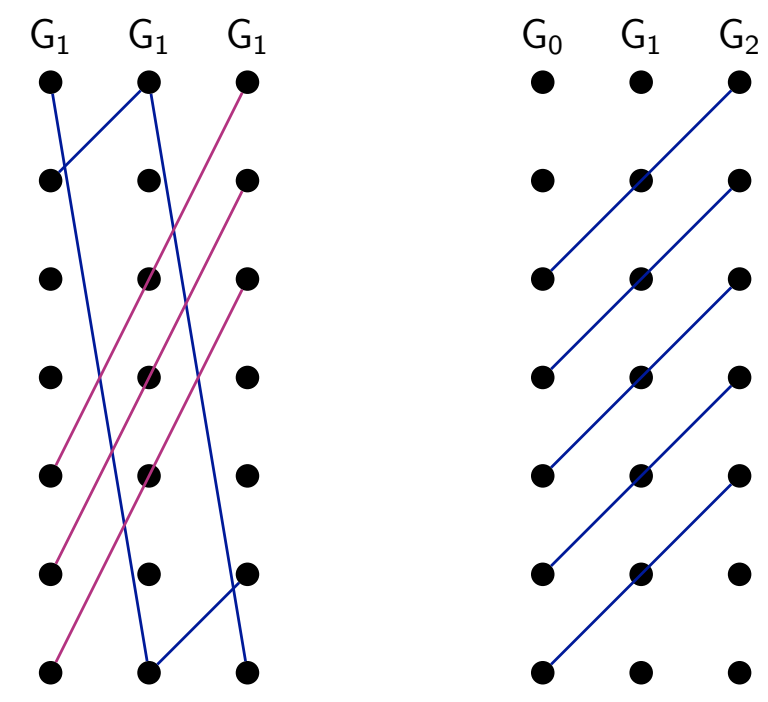

Figure 2.5: Partial parallel classes of size 5

Lemma 19 [10] A 3-RGDD $\left(g^{u}\right)$ exists if and only if $u \geq 3, g(u-1)$ is even, $g u \equiv 0$ $(\bmod 3)$.

Lemma 20 There exists an $S T S_{8}(33)$.

\section{Proof}

The four groups of size 8 are:

$G_{0}=\left\{1_{0}, 2_{0}, 3_{0}, 4_{0}, 5_{0}, 6_{0}, 7_{0}, 8_{0}\right\}, G_{1}=\left\{1_{1}, 2_{1}, 3_{1}, 4_{1}, 5_{1}, 6_{1}, 7_{1}, 8_{1}\right\}$, $G_{2}=\left\{1_{2}, 2_{2}, 3_{2}, 4_{2}, 5_{2}, 6_{2}, 7_{2}, 8_{2}\right\}$, and $G_{3}=\left\{1_{3}, 2_{3}, 3_{3}, 4_{3}, 5_{3}, 6_{3}, 7_{3}, 8_{3}\right\}$. The elements of these groups will be the points of the STS $(33)$. The 22 partial parallel classes of size 8 are as follows.

\begin{tabular}{|c|c|c|c|c|c|c|c|c|c|c|}
\hline \multicolumn{2}{|c|}{$P P C 1$} & \multicolumn{3}{|c|}{$P P C 2$} & \multicolumn{3}{|c|}{$P P C 3$} & \multicolumn{3}{|c|}{ PPC4 } \\
\hline $7_{0} \quad 8_{0}$ & $\infty$ & $3_{0}$ & $6_{0}$ & $\infty$ & $1_{0}$ & $6_{2}$ & $6_{3}$ & $1_{0}$ & $5_{2}$ & $5_{3}$ \\
\hline $2_{1}$ & $8_{3}$ & $6_{1}$ & $6_{2}$ & $4_{3}$ & $2_{0}$ & $5_{2}$ & $7_{3}$ & $2_{0}$ & $6_{2}$ & $8_{3}$ \\
\hline $3_{1}$ & $6_{3}$ & $7_{1}$ & $7_{2}$ & $2_{3}$ & $3_{0}$ & $8_{2}$ & $5_{3}$ & $3_{0}$ & $7_{2}$ & $6_{3}$ \\
\hline $4_{1}$ & $7_{3}$ & $8_{1}$ & $8_{2}$ & $3_{3}$ & $7_{1}$ & $8_{1}$ & $\infty$ & $4_{0}$ & $8_{2}$ & $7_{3}$ \\
\hline $5_{1}$ & $2_{3}$ & $1_{1}$ & $2_{2}$ & $6_{3}$ & $5_{0}$ & $1_{2}$ & $1_{3}$ & $5_{0}$ & $2_{2}$ & $2_{3}$ \\
\hline $6_{1}$ & $3_{3}$ & $2_{1}$ & $1_{2}$ & $7_{3}$ & $6_{0}$ & $2_{2}$ & $4_{3}$ & $6_{0}$ & $1_{2}$ & $3_{3}$ \\
\hline $7_{1}$ & $1_{3}$ & $3_{1}$ & $4_{2}$ & $5_{3}$ & $7_{0}$ & $3_{2}$ & $2_{3}$ & $7_{0}$ & $4_{2}$ & $1_{3}$ \\
\hline $8_{1}$ & $4_{3}$ & $4_{1}$ & $3_{2}$ & $8_{3}$ & $8_{0}$ & $4_{2}$ & $3_{3}$ & $3_{1}$ & $6_{1}$ & $\infty$ \\
\hline
\end{tabular}




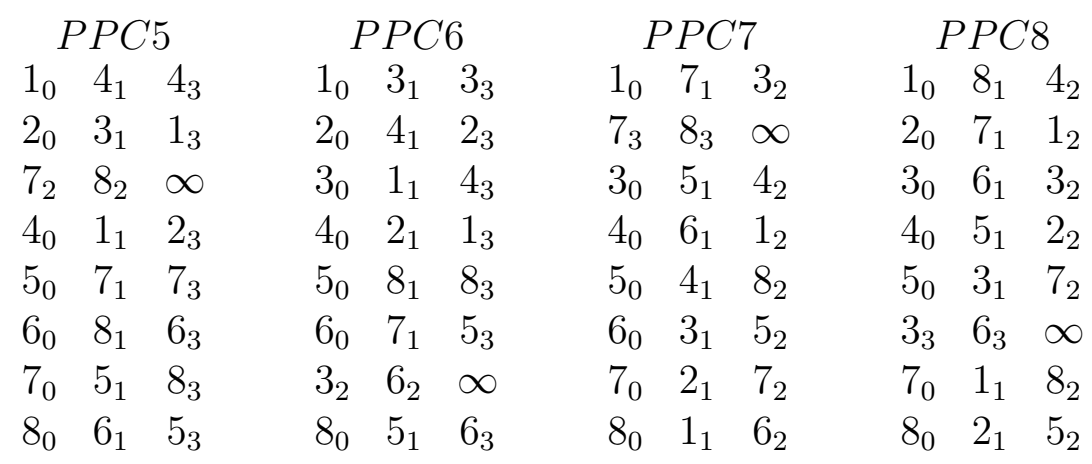

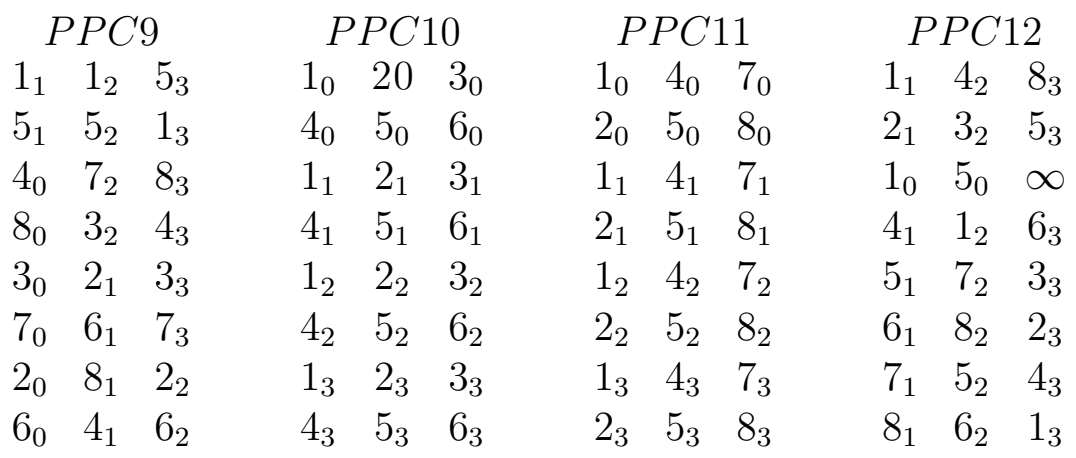

\begin{tabular}{|c|c|c|c|c|c|c|c|c|c|c|}
\hline \multicolumn{2}{|c|}{$P P C 13$} & \multicolumn{3}{|c|}{ PPC14 } & \multicolumn{3}{|c|}{ PPC15 } & \multicolumn{3}{|c|}{ PPC16 } \\
\hline $1_{1} \quad 3_{2}$ & $7_{3}$ & $1_{0}$ & $7_{2}$ & $7_{3}$ & $1_{0}$ & $8_{2}$ & $8_{3}$ & $1_{2}$ & $5_{2}$ & $\infty$ \\
\hline $2_{1}$ & $6_{3}$ & $1_{1}$ & $5_{1}$ & $\infty$ & $2_{0}$ & $7_{2}$ & $5_{3}$ & $2_{0}$ & $2_{1}$ & $4_{3}$ \\
\hline $3_{1}$ & $8_{3}$ & $3_{0}$ & $5_{2}$ & $8_{3}$ & $3_{0}$ & $6_{2}$ & $7_{3}$ & $3_{0}$ & $3_{1}$ & $2_{3}$ \\
\hline $4_{1}$ & $5_{3}$ & $4_{0}$ & $6_{2}$ & $5_{3}$ & $4_{0}$ & $5_{2}$ & $6_{3}$ & $4_{0}$ & $4_{1}$ & $3_{3}$ \\
\hline $5_{1}$ & $4_{3}$ & $5_{0}$ & $4_{2}$ & $4_{3}$ & $5_{0}$ & $3_{2}$ & $3_{3}$ & $5_{0}$ & $6_{1}$ & $6_{3}$ \\
\hline $6_{1}$ & $1_{3}$ & $6_{0}$ & $3_{2}$ & $1_{3}$ & $2_{1}$ & $4_{1}$ & $\infty$ & $6_{0}$ & $5_{1}$ & $7_{3}$ \\
\hline $2_{0}$ & $\infty$ & $7_{0}$ & $2_{2}$ & $3_{3}$ & $7_{0}$ & $1_{2}$ & $4_{3}$ & $7_{0}$ & $8_{1}$ & $5_{3}$ \\
\hline $8_{1}$ & $2_{3}$ & $8_{0}$ & $1_{2}$ & $2_{3}$ & $8_{0}$ & $2_{2}$ & $1_{3}$ & $8_{0}$ & $7_{1}$ & $8_{3}$ \\
\hline
\end{tabular}




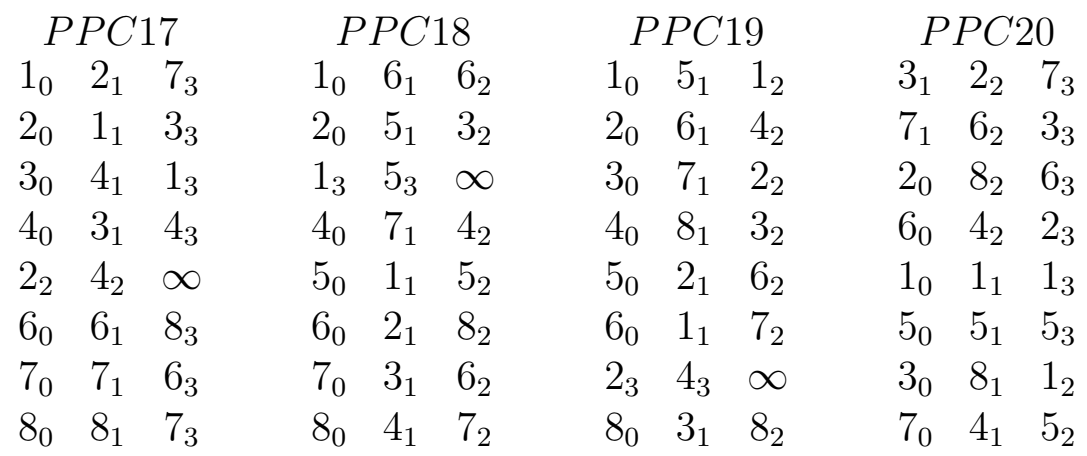

\begin{tabular}{ccccccc}
\multicolumn{3}{c}{$P P C 21$} & \multicolumn{4}{c}{$P P C 22$} \\
$2_{0}$ & $6_{0}$ & $7_{0}$ & & $1_{0}$ & $6_{0}$ & $8_{0}$ \\
$3_{0}$ & $4_{0}$ & $8_{0}$ & & $3_{0}$ & $5_{0}$ & $7_{0}$ \\
$2_{1}$ & $6_{1}$ & $7_{1}$ & & $1_{1}$ & $6_{1}$ & $8_{1}$ \\
$3_{1}$ & $4_{1}$ & $8_{1}$ & & $3_{1}$ & $5_{1}$ & $7_{1}$ \\
$2_{2}$ & $6_{2}$ & $7_{2}$ & & $1_{2}$ & $6_{2}$ & $8_{2}$ \\
$3_{2}$ & $4_{2}$ & $8_{2}$ & $3_{2}$ & $5_{2}$ & $7_{2}$ \\
$2_{3}$ & $6_{3}$ & $7_{3}$ & & $1_{3}$ & $6_{3}$ & $8_{3}$ \\
$3_{3}$ & $4_{3}$ & $8_{3}$ & $3_{3}$ & $5_{3}$ & $7_{3}$
\end{tabular}

The following discussion describes the procedure used to obtain the decomposition given in Lemma 20.

An $S T S(33)$ has 176 blocks. If we want to partition the blocks into partial parallel classes of size 8 , then we will have 22 such classes.

In order to do that we start with a 3 -frame of type $2^{4}$, which we know exists from Lemma 13. A 3-frame of type $2^{4}$ was given previously in Example 1.1.3. We illustrate it again in Figure 2.6. We give each point of this frame weight 4 , so we will have 4 groups of size 8 and we add to it the $\{\infty\}$ point, so we get in total 33 points. Then we use a resolvable group divisible design, $R G D D\left(4^{3}\right)$ on each of the blocks of the 3 -frame. The frame parallel classes will tell us which groups to use to get the blocks of the partial parallel classes. The first frame parallel class indicates us that we are not going to use $G_{0}$ and so on. When we miss $G_{0}$, we join to it the $\{\infty\}$ point and put on it a $K T S(9)$. And we do the same when we miss the other three groups $G_{1}, G_{2}, G_{3}$. 
Since we gave to each point weight 4 , then each block of the frame would give us 12 points, so we put an $R G D D\left(4^{3}\right)$ on these points. Without loss of generality we assume that parallel class 1, (PC1) of the $R G D D\left(4^{3}\right)$ looks like in Figure 2.6. So we consider this parallel class on the $R G D D\left(4^{3}\right)$ that we put on block $1,\{3,5,8\}$ of frame parallel class 1 , (FPC1). On the $R G D D\left(4^{3}\right)$ that we put on block $2,\{4,6,7\}$ of frame parallel class 1, (FPC1) we take in consideration parallel class 2, (PC2). We take out one block from PC1. The block we take out is given in red in Figure 2.7, and we replace it with the block that contains the $\{\infty\}$ point on PC1 of the $K T S(9)$ that we put on $G_{0} \cup\{\infty\}$. This other block is given in green in Figure 2.7. This gives us 8 blocks, since we used two parallel classes of two $R G D D s\left(4^{3}\right)$. So we have constructed the first $8-P P C$ of an $S T S(33)$.

We repeat the same procedure again in these two blocks of FPC1, but now on the $R G D D\left(4^{3}\right)$ that we put on block 1, we work with PC2, and on the $R G D D\left(4^{3}\right)$ that we put on block 2, we work with PC1. Again we take out one block from PC1. This block is given in red in Figure 2.8, and we replace it with the block that contains the $\{\infty\}$ point on PC2 of the KTS(9). This block is the one given in green color on 2.7. In this way we get another $8-P P C$.

On FPC2 we miss $G_{1}$. On $G_{1} \cup\{\infty\}$ we put a $K T S(9)$. Again we put an $R G D D\left(4^{3}\right)$ on block $1,\{1,6,8\}$ and one other on block $2,\{2,5,7\}$ of this frame parallel class. We consider on block $1, \mathrm{PC} 2$ of the $R G D D\left(4^{3}\right)$, and on block 2 , PC1 of the $R G D D\left(4^{3}\right)$. We take out the red block in Figure 2.8 from PC2, and we replace it with the green block that contains the $\{\infty\}$ point on PC1 of the KTS(9). Thus we have get PPC3 of STS $(33)$.

We continue to work on these two blocks of FPC2. Now we change the parallel classes that we consider on the $R G D D s\left(4^{3}\right)$ that we put on them. On block 1, we consider PC1 and on block 2, PC2. Again we take out one block from PC2, the red block in Figure 2.8, and replace it with the green block that contains the $\{\infty\}$ point on PC2 of the KTS(9). This counts for the fourth $8-P P C$ of the STS(33).

The same procedure is followed when working on FPC3 and FPC4. In these two last frames, we work with PC3 and PC4 of the $R G D D s\left(4^{3}\right)$ that we put on their respective blocks.

As we can see from each frame parallel class we get 2 partial parallel classes of size 8 . From all the 4 frame parallel classes we get 8 partial parallel classes of size 8 . This is illustrated Figures 2.8, 2.9, and 2.10. Another partial parallel class is created with all the red blocks that we took out from the $R G D D s\left(4^{3}\right)$ before. This is illustrated in 
Figure 2.11. Now the number of $8-P P C s$ is 9.

The blocks we took out from the $R G D D s\left(4^{3}\right)$ were replaced with blocks from the $K T S(9)$ (the green blocks). In each parallel class of a $K T S(9)$ there are 3 blocks. Taking out one block we are left with 2. So we have 2 blocks left in PC1 and 2 blocks left in PC2 of the KTS(9) that we used on $G_{0}$, which was the group that was missed by FPC1. Each frame parallel class gives us these 4 blocks of the $K T S(9)$, and furthermore none of these blocks contains $\{\infty\}$. Thus we may take the 2 leftover blocks on $G_{0}, G_{1}, G_{2}$, and $G_{3}$ from PC1 to make a partial parallel class of size 8 . We may do the same with the left over blocks from PC2 to make another partial parallel class. So far we have in total $118-P P C s$.

As we mentioned above we need to have $228-P P C s$ all together. To construct the 11 partial parallel classes we used only PC1 and PC2 of the $R G D D s\left(4^{3}\right)$ we put on the blocks of FPC1 and FPC2. On FPC3 and FPC4 we used only PC3 and PC4 of $R G D D s\left(4^{3}\right)$ that we out on their blocks. On all the FPCs we used PCI and PC2 of the $K T S(9)$. Now on the $R G D D s\left(4^{3}\right)$ that we put on the blocks of FPC1 and FPC2 we consider PC3 and PC4. On the $R G D D s\left(4^{3}\right)$ that we put on the blocks of FPC3 and FPC4 we consider PC1 and PC2. This time on all the frame parallel classes we consider PC3 and PC4 of the KTS(9). This we get 8 more $8-P P C s$ from the frame parallel classes. We get one more from the blocks we take out from parallel classes of the $R G D D s\left(4^{3}\right)$. Two other $8-P P C s$ are obtained from the left over block on PC3 and PC4 of the KTS(9). Hence 11 more partial parallel classes of size 8 . This completes the $228-P P C s$ of the $S T S(33)$.

We illustrate the whole process of constructing the partial parallel classes in Figures 2.7 -2.16 . 

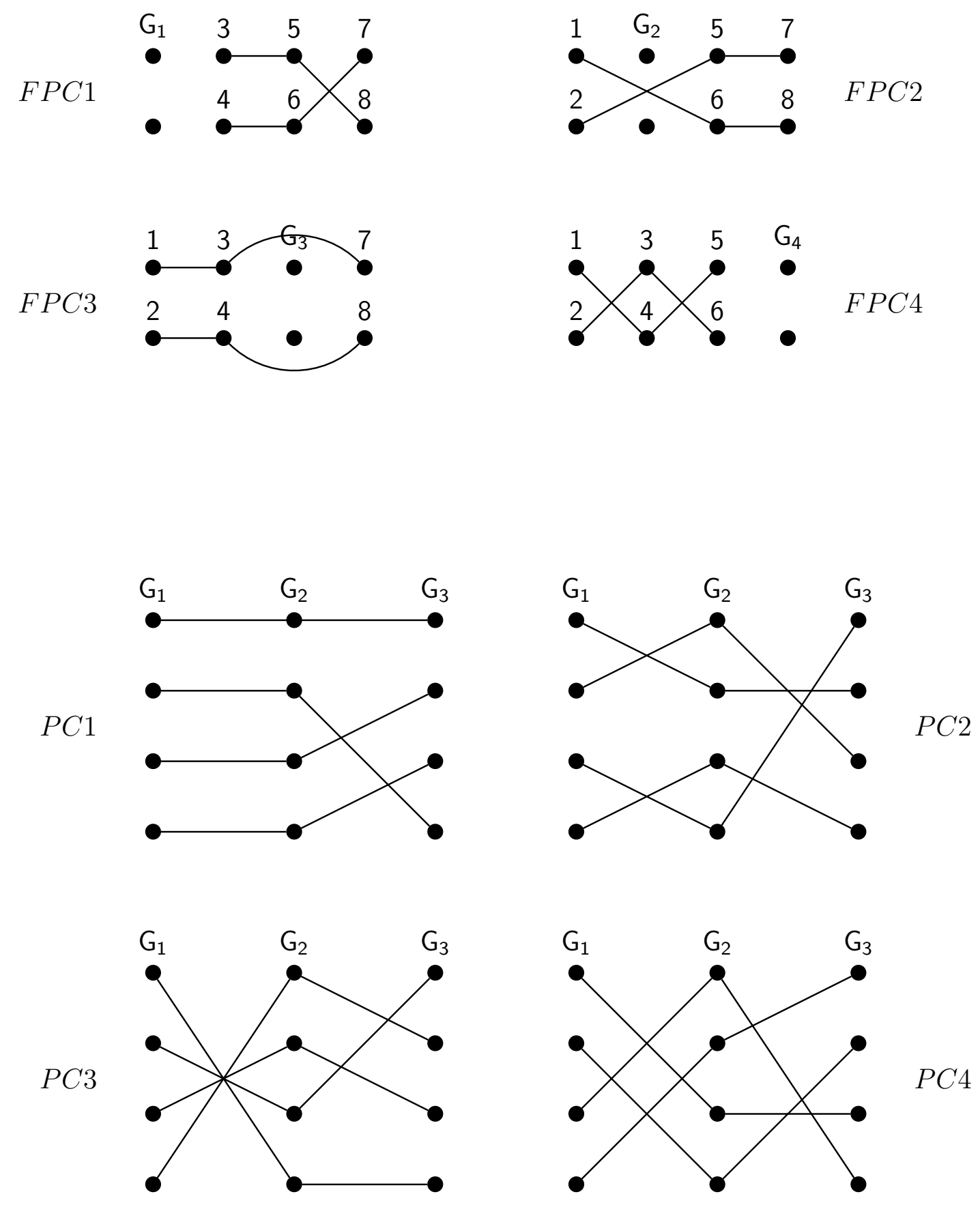

Figure 2.6: 3-frame of type $2^{4}$ and an $R G D D\left(4^{3}\right)$ 

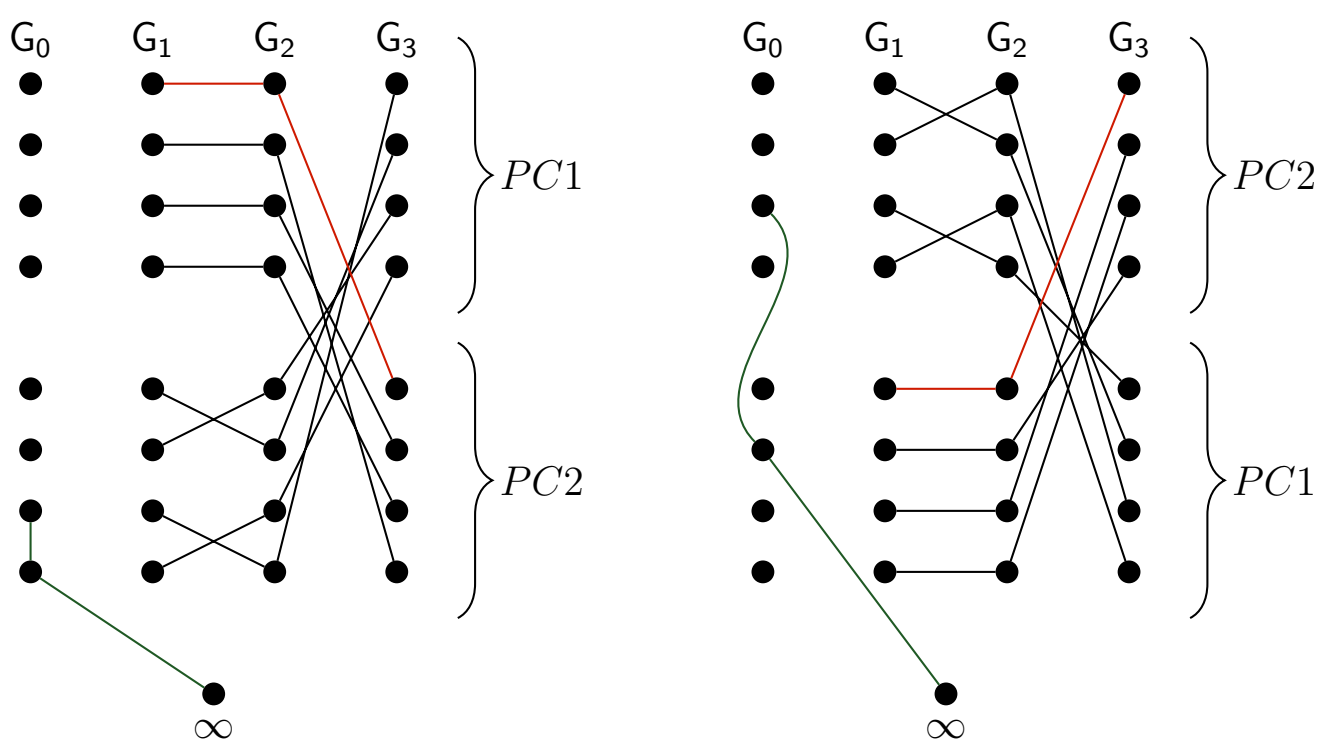

Figure 2.7: Two 8-PPC's using FPC1
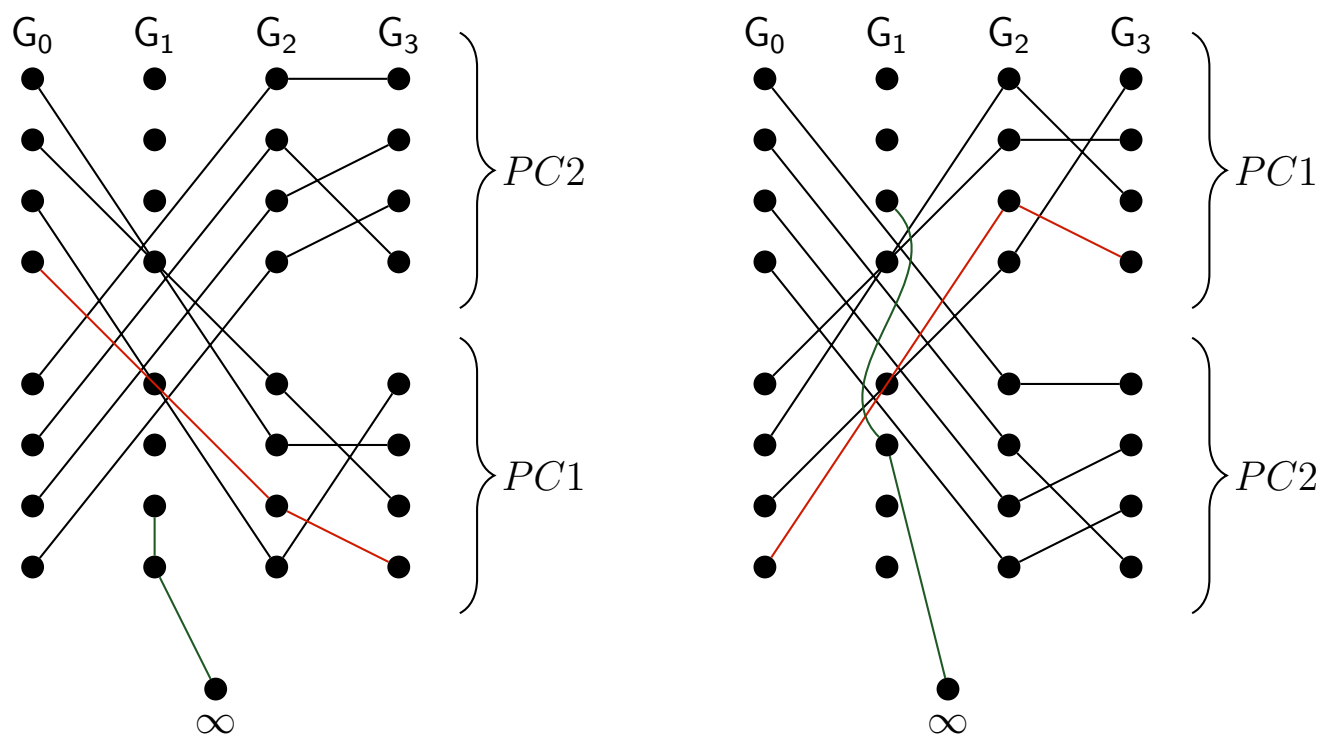

Figure 2.8: Two 8-PPC's using FPC2 

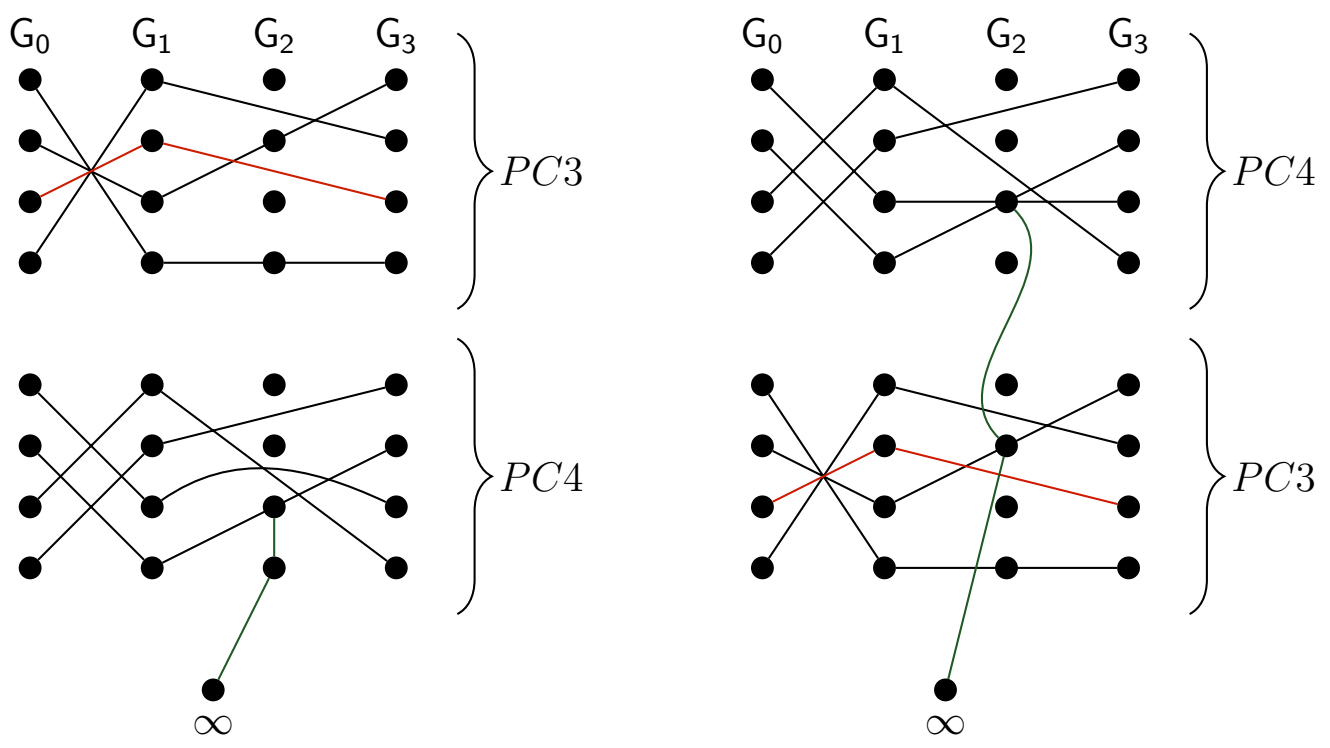

Figure 2.9: Two 8-PPC's using FPC3
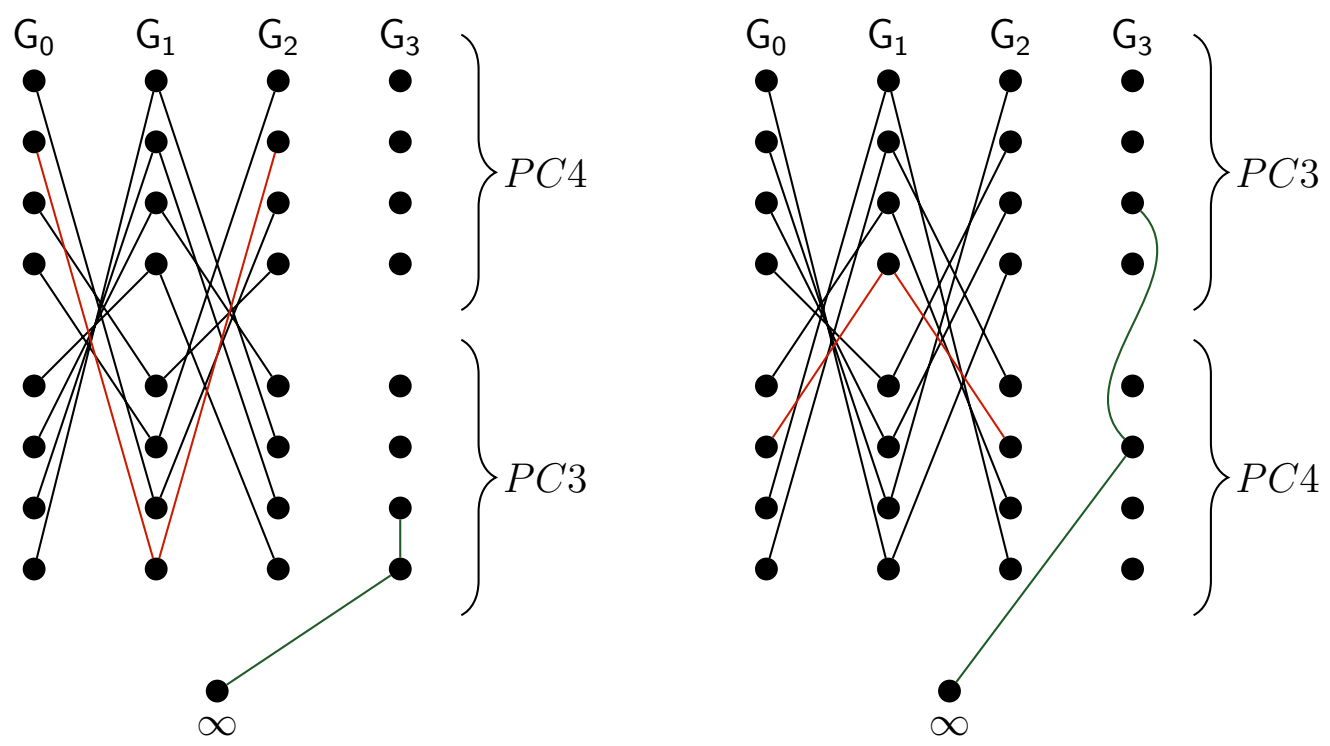

Figure 2.10: Two 8-PPC's using FPC4 


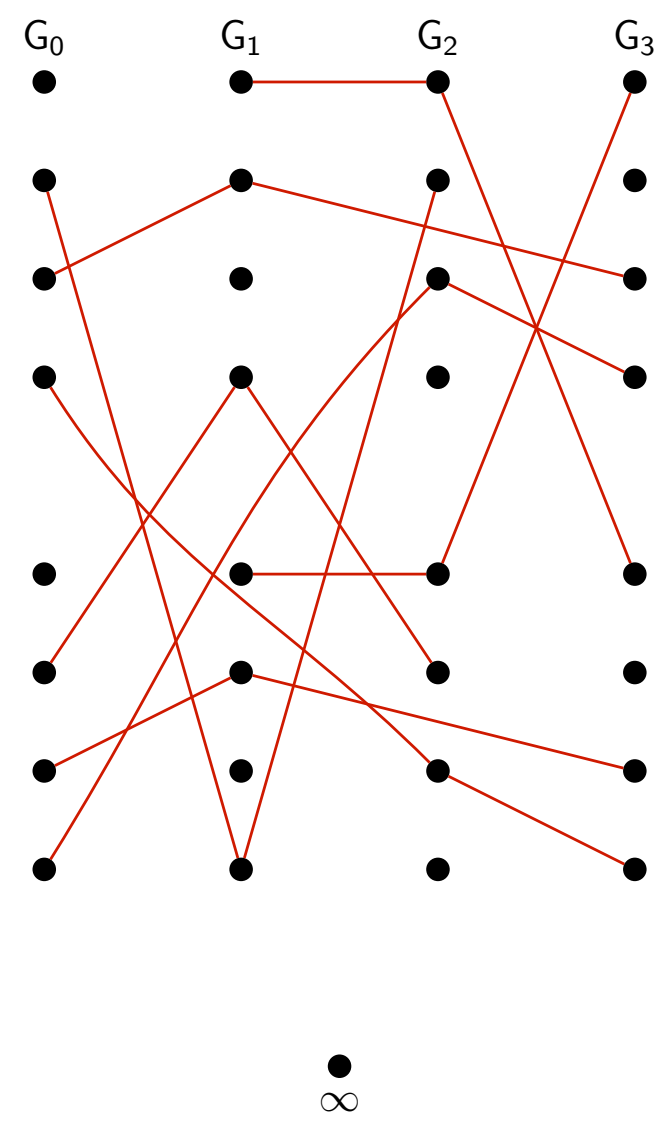

Figure 2.11: The 8-PPC created by all the blocks that we took out from the RGDD's. 

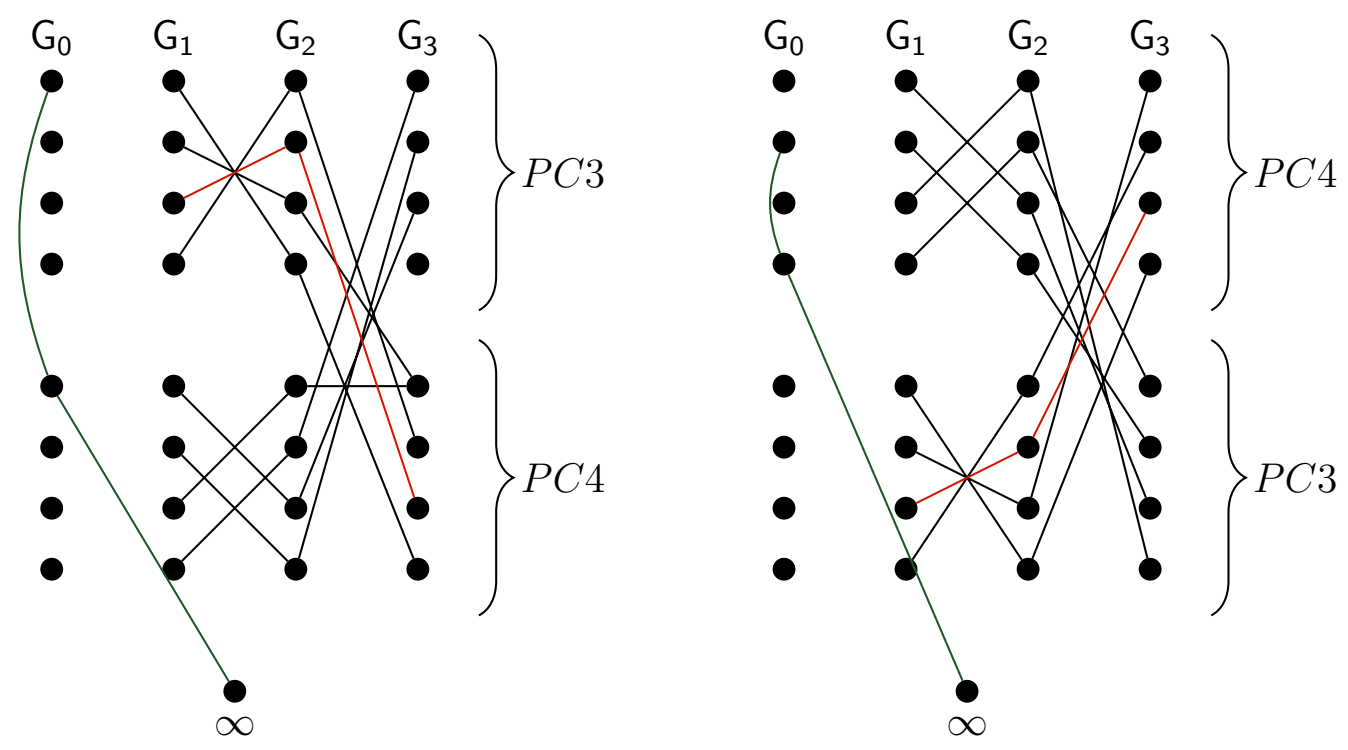

Figure 2.12: Two 8-PPC's using FPC1
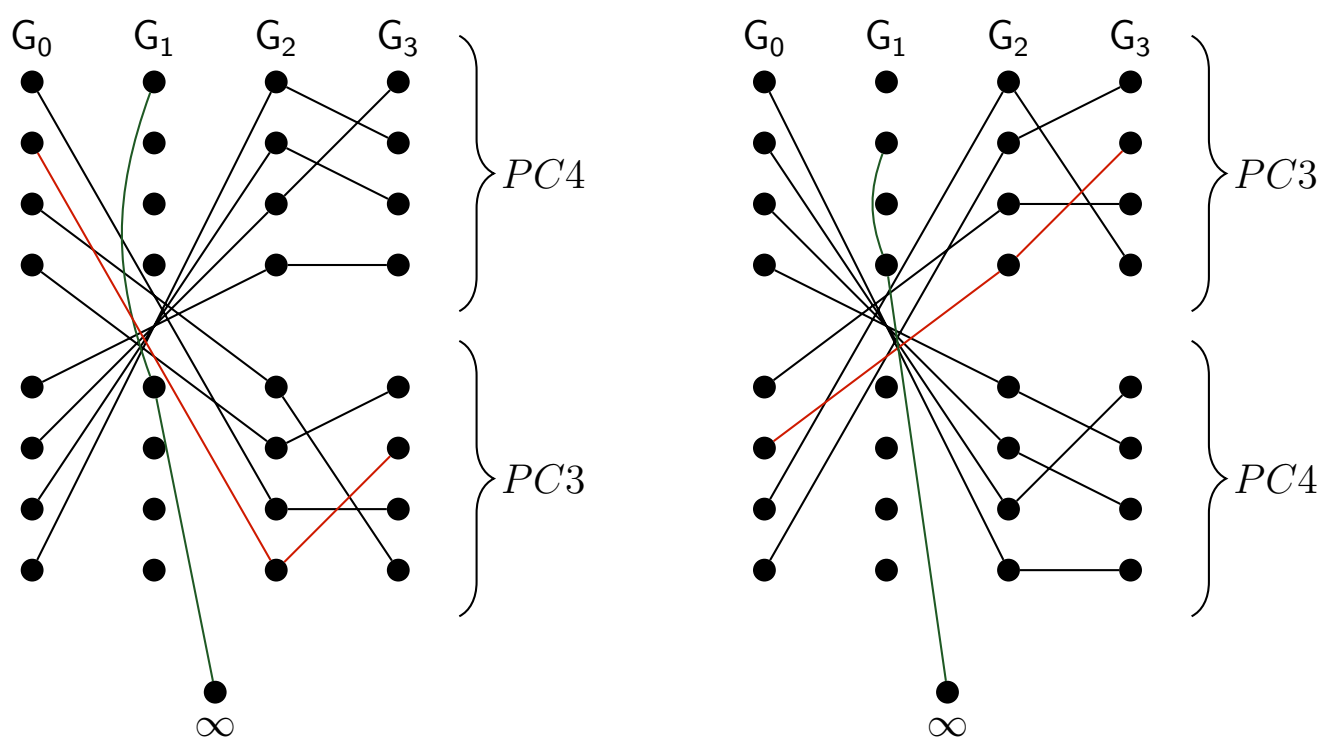

Figure 2.13: Two 8-PPC's using FPC2 

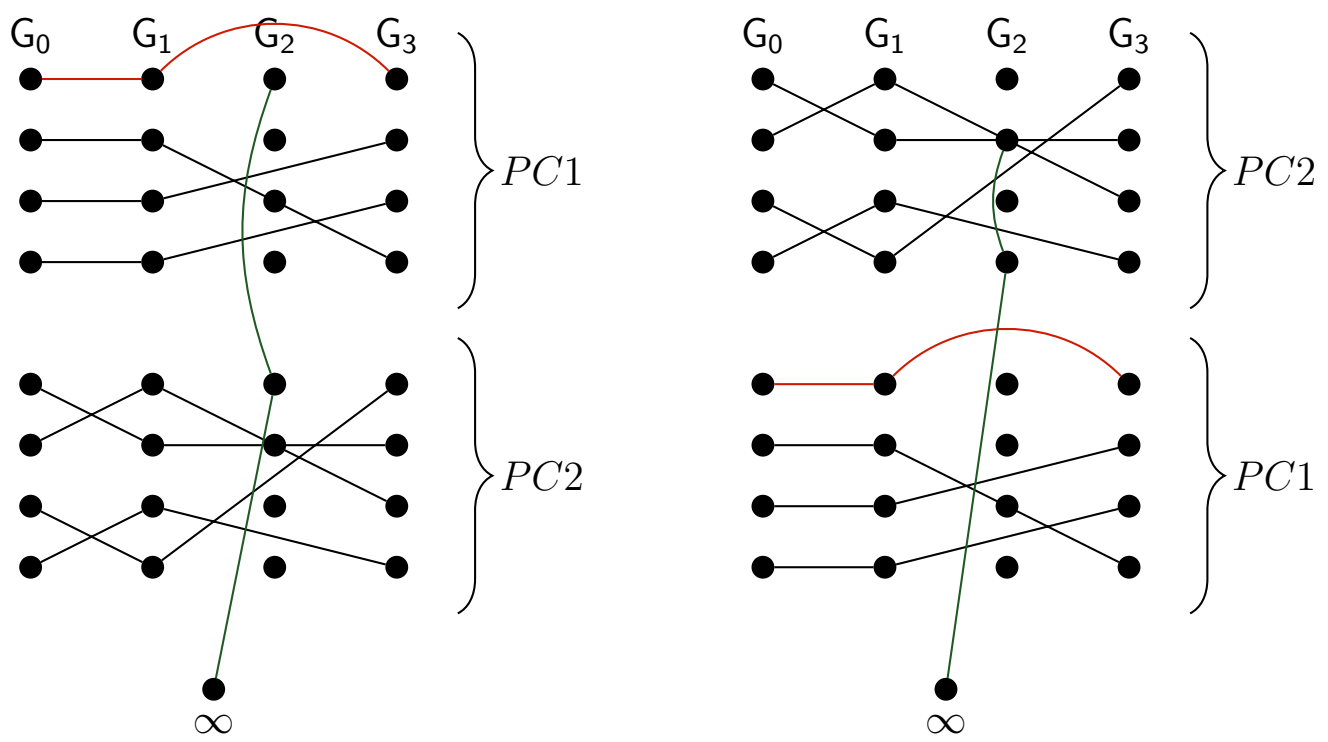

Figure 2.14: Two 8-PPC's using FPC3
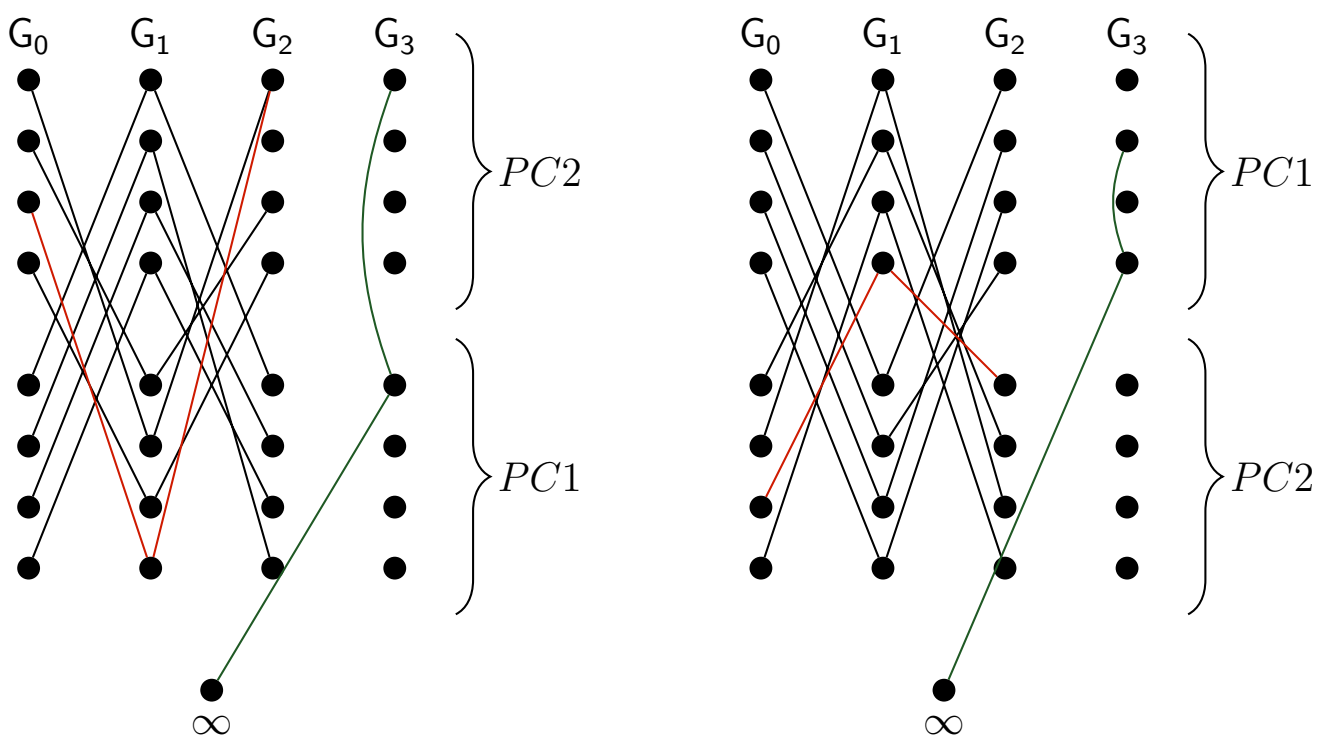

Figure 2.15: Two 8-PPC's using FPC4 


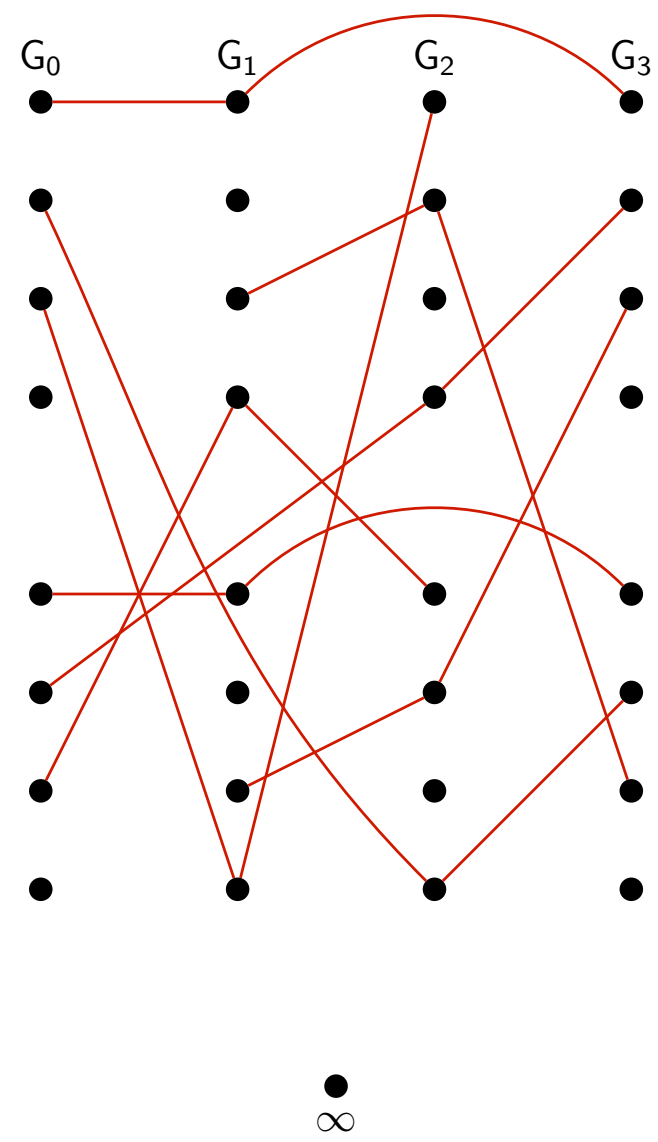

Figure 2.16: The 8-PPC created by all the blocks that we took out from the RGDD's. 


\section{Chapter 3}

\section{Summary, Conclusions and Further Research}

\subsection{Summary and Conclusions}

In this report we studied the problem of decomposing Steiner triple systems into partial parallel classes of size $m$. We developed some constructions on how to solve this problem, namely the constructions in Lemma 14 and in Lemma 16 . As in the paper [1] that motivated our study, we wanted to cover the cases $9 \leq v \leq 32$. We extended the order of the Steiner triple systems to $v=43$. For orders $9 \leq v \leq 43$ we found ways to partition the triples into partial parallel classes of size $m$. So every time $v \equiv 3$ $(\bmod 6)$ and the number of blocks is even we can apply Lemma 14 to obtain partial parallel classes of size 2 . And equivalently for partial parallel classes of size 4 , when $v \equiv 3(\bmod 6)$ and the number of blocks is divisible by 4 we use Lemma 16 .

We obtain some other partitions into partial parallel classes by using already known results. For example we use Kirkman triple systems and Hanani triple systems to obtain the partitions in many cases.

There were three cases that we treated separately and we used different methods.

For the Steiner triple system of order 21, to partition it into partial parallel classes of size 5 we used a Steiner triple system of order 7 in each of its three 7-points. We worked $(\bmod 7)$ and so we covered all the differences the same number of times.

To construct the 20 partial parallel classes of size 5 of a $S T S(25)$ we used the construction described by Lemma 12 given in [1].

The last case was $S T S(33)$ and its size 8 partial parallel classes. As we described in Chapter 2 in this case we used initially a 3 -frame of type $2^{4}$. Giving weight 4 to each point of this frame and adding another point $\{\infty\}$, we had the necessary points to con- 
struct the 22 partial parallel classes of size 8 of the $S T S(33)$. Then we needed to use an $R G D D\left(4^{3}\right)$, and we also needed the use of a $K T S(9)$.

Hence because we were able to construct the partial parallel classes mentioned above, we conclude that there exist an $S T S_{5}(21)$, an $S T S_{5}(25)$, and an $S T S_{8}(33)$.

As a brief partial summary of the results in this report we have the following theorems.

Theorem 21 If $2 \mid b$, then there exists an $S T S(v)$ whose blocks can be partitioned into $\frac{b}{2} 2-P P C s$.

Theorem 22 If $4 \mid b$, then there exists an $S T S(v)$ whose blocks can be partitioned into $\frac{b}{4} 4-P P C s$.

The tables below, Table 3.1, 3.2, and 3.3 summarize our results, and give the current state of the problem up to $v=99$. Up to $v=43$ we could partition them for each value of $m$. After this order we used the constructions mentioned above to cover the partitions for some of the $m$ values. New results in the summary tables are marked with an $(*)$.

\subsection{Future work}

The methods used in Lemmas 14, and 16 could possibly be generalized. These two lemmas take care of cases when $m=2$ and $m=4$. We conjecture that this can always be done for large values of $m$, when some necessary conditions are met. The method used for the case of $v=33$ and $m=8$ also shows promise for generalization. 
Table 3.1: Summary table

\begin{tabular}{|c|c|c|c|c|}
\hline$v$ & $b$ & $\left.\frac{v}{3}\right\rfloor$ & $m$ & Construction \\
\hline \multirow[t]{2}{*}{9} & \multirow[t]{2}{*}{12} & \multirow[t]{2}{*}{3} & 2 & Theorem 10 \\
\hline & & & 3 & Lemma \\
\hline 13 & 26 & 4 & 2 & Lemma 12 \\
\hline 15 & 35 & 5 & 5 & Lemma 6 \\
\hline 19 & 54 & 6 & 3 & Lemma 8 \\
\hline \multirow[t]{3}{*}{21} & \multirow[t]{3}{*}{70} & \multirow[t]{3}{*}{7} & 2 & * Lemma 14 \\
\hline & & & 5 & * Lemma $\overline{\overline{18}}$ \\
\hline & & & 7 & Lemma 6 \\
\hline \multirow[t]{3}{*}{25} & \multirow[t]{3}{*}{100} & \multirow[t]{3}{*}{8} & 2 & Lemma \\
\hline & & & 4 & Lemma 8 \\
\hline & & & 5 & Lemma 12 \\
\hline \multirow[t]{2}{*}{27} & \multirow[t]{2}{*}{117} & \multirow[t]{2}{*}{9} & 3 & Lemma 7 \\
\hline & & & 9 & Lemma 6 \\
\hline 31 & 155 & 10 & 5 & Lemma 8 \\
\hline \multirow[t]{4}{*}{33} & \multirow[t]{4}{*}{176} & \multirow[t]{4}{*}{11} & 2 & * Lemma 14 \\
\hline & & & 4 & * Lemma $\overline{\overline{16}}$ \\
\hline & & & 8 & * Lemma $\overline{20}$ \\
\hline & & & 11 & Lemma 6 \\
\hline \multirow[t]{2}{*}{37} & \multirow[t]{2}{*}{222} & \multirow[t]{2}{*}{12} & 2 & Lemma 8 \\
\hline & & & 3 & Lemma 8 \\
\hline 39 & 247 & 13 & 13 & Lemma 6 \\
\hline 43 & 301 & 14 & 7 & Lemma 8 \\
\hline \multirow[t]{7}{*}{45} & \multirow[t]{7}{*}{330} & \multirow[t]{7}{*}{15} & 2 & * Lemma 14 \\
\hline & & & 3 & Lemma \\
\hline & & & 5 & Lemma 7 \\
\hline & & & 6 & $?$ \\
\hline & & & 10 & $?$ \\
\hline & & & 11 & $?$ \\
\hline & & & 15 & Lemma 6 \\
\hline \multirow[t]{5}{*}{49} & \multirow[t]{5}{*}{392} & \multirow[t]{5}{*}{16} & 2 & Lemma 8 \\
\hline & & & 4 & Lemma 8 \\
\hline & & & 7 & $?$ \\
\hline & & & 8 & Lemma 8 \\
\hline & & & 14 & $?$ \\
\hline
\end{tabular}


Table 3.2: Summary table

\begin{tabular}{|c|c|c|c|c|}
\hline$v$ & $b$ & $\frac{v}{3}$ & $m$ & Construction \\
\hline \multirow[t]{2}{*}{51} & \multirow[t]{2}{*}{425} & \multirow[t]{2}{*}{17} & 5 & $?$ \\
\hline & & & 17 & Lemma 6 \\
\hline \multirow[t]{5}{*}{55} & \multirow[t]{5}{*}{495} & \multirow[t]{5}{*}{18} & 3 & Lemma $\overline{8}$ \\
\hline & & & 5 & Theorem 9 \\
\hline & & & 9 & Lemma 8 \\
\hline & & & 11 & $?$ \\
\hline & & & 15 & $?$ \\
\hline \multirow[t]{5}{*}{57} & \multirow[t]{5}{*}{532} & \multirow[t]{5}{*}{19} & 2 & * Lemma 14 \\
\hline & & & 4 & * Lemma 16 \\
\hline & & & 7 & $?$ \\
\hline & & & 14 & $?$ \\
\hline & & & 19 & Lemma 6 \\
\hline \multirow[t]{3}{*}{61} & \multirow[t]{3}{*}{610} & \multirow[t]{3}{*}{20} & 2 & Lemma 8 \\
\hline & & & 5 & Lemma 8 \\
\hline & & & 10 & Lemma 8 \\
\hline \multirow[t]{3}{*}{63} & \multirow[t]{3}{*}{651} & \multirow[t]{3}{*}{21} & 3 & Lemma 7 \\
\hline & & & 7 & Lemma 7 \\
\hline & & & 21 & Lemma 6 \\
\hline 67 & 737 & 22 & 11 & Lemma 8 \\
\hline \multirow[t]{3}{*}{69} & \multirow[t]{3}{*}{782} & \multirow[t]{3}{*}{23} & 2 & * Lemma 14 \\
\hline & & & 17 & $?$ \\
\hline & & & 23 & Lemma 6 \\
\hline \multirow[t]{5}{*}{73} & \multirow[t]{5}{*}{876} & \multirow[t]{5}{*}{24} & 2 & Lemma 8 \\
\hline & & & 3 & Lemma 8 \\
\hline & & & 4 & Lemma 8 \\
\hline & & & 6 & Lemma 8 \\
\hline & & & 12 & Lemma 8 \\
\hline \multirow[t]{2}{*}{75} & \multirow[t]{2}{*}{925} & \multirow[t]{2}{*}{25} & 5 & Lemma 7 \\
\hline & & & 25 & Lemma 6 \\
\hline 79 & 1027 & 26 & 13 & Lemma 8 \\
\hline \multirow[t]{6}{*}{81} & & & 12 & $?$ \\
\hline & & & 15 & $?$ \\
\hline & & & 18 & $?$ \\
\hline & & & 20 & $?$ \\
\hline & & & 24 & $?$ \\
\hline & & & 27 & Lemma 6 \\
\hline
\end{tabular}


Table 3.3: Summary table

\begin{tabular}{|c|c|c|c|c|}
\hline$v$ & $b$ & $\left\lfloor\frac{v}{3}\right\rfloor$ & $m$ & Construction \\
\hline \multirow[t]{8}{*}{81} & \multirow[t]{8}{*}{1080} & \multirow[t]{8}{*}{27} & 2 & * Lemma 14 \\
\hline & & & 3 & Lemma 7 \\
\hline & & & 4 & * Lemma 16 \\
\hline & & & 5 & $?$ \\
\hline & & & 6 & $?$ \\
\hline & & & 8 & $?$ \\
\hline & & & 9 & Lemma 7 \\
\hline & & & 10 & $?$ \\
\hline \multirow[t]{6}{*}{85} & \multirow[t]{6}{*}{1190} & \multirow[t]{6}{*}{28} & 2 & Lemma 8 \\
\hline & & & 5 & Theorem 9 \\
\hline & & & 7 & Lemma 8 \\
\hline & & & 10 & $?$ \\
\hline & & & 14 & Lemma 8 \\
\hline & & & 17 & $?$ \\
\hline 87 & 1247 & 29 & 29 & Lemma 6 \\
\hline \multirow[t]{6}{*}{91} & \multirow[t]{4}{*}{1365} & \multirow[t]{4}{*}{30} & 3 & Lemma 8 \\
\hline & & & 5 & Lemma 8 \\
\hline & & & 7 & Theorem 9 \\
\hline & & & 13 & $?$ \\
\hline & \multirow[t]{2}{*}{1365} & \multirow[t]{2}{*}{30} & 15 & Lemma 8 \\
\hline & & & 21 & $?$ \\
\hline \multirow[t]{3}{*}{93} & \multirow[t]{3}{*}{1426} & \multirow[t]{3}{*}{31} & 2 & * Lemma 14 \\
\hline & & & 23 & $?$ \\
\hline & & & 31 & Lemma 6 \\
\hline \multirow[t]{4}{*}{97} & \multirow[t]{4}{*}{1552} & \multirow[t]{4}{*}{32} & 2 & Lemma 8 \\
\hline & & & 4 & Lemma 8 \\
\hline & & & 8 & Lemma 8 \\
\hline & & & 16 & Lemma 8 \\
\hline \multirow[t]{5}{*}{99} & \multirow[t]{5}{*}{1617} & \multirow[t]{5}{*}{33} & 3 & Lemma 7 \\
\hline & & & 7 & Theorem 9 \\
\hline & & & 11 & Lemma 7 \\
\hline & & & 21 & $?$ \\
\hline & & & 33 & Lemma 6 \\
\hline
\end{tabular}




\section{Bibliography}

[1] C.J. Colbourn, D.Horsley and C.Wang, Colouring Triples in Every Way: A Conjecture, Quaderni di Matematica, 28 (2012), 257-286.

[2] D.R. Stinson, Frames for Kirkman triple system, Discrete Math. 65 (1987), 289300.

[3] P. Horák, A. Rosa, Decomposing Steiner Triple Systems into Small Configurations, Ars Combinatoria, 26 (1988), 91-105.

[4] D.K. Ray-Chaudhuri and R.M. Wilson, Solution of Kirkman's school-girl problem, Proc. Sympos. Pure Math. 19 (1971), Amer. Math. Soc., 187-203.

[5] D.K. Ray-Chaudhuri and R.M. Wilson, The existence of resolvable block designs, in A Survey of Combinatorial Theory (Ed.J.N. Srivastava), North-Holland, Amsterdam (1975), 361-375.

[6] S.A. Vanstone, D.R. Stinson, P.J. Schellenberg, A. Rosa, R. Rees, C.J. Colbourn, M.W. Carter, and J.E. Carter, Hanani Triple Systems, Israel J. Math. 83 (1993), 305-319.

[7] T.P. Kirkman, On a problem in Combinations, Cambridge and Dublin Math J. 2 (1847), 191-204. (12,15,59).

[8] J. Schönheim, On maximal systems of k-tuples, Studia Sci. Math. Hungar. 1(1996), 363-368.

[9] C.J. Colbourn and S. Zhao, Maximum Kirkman signal sets for synchronous unipolar multi-user communications systems, Des. Codes Cryptogr. 20 (200), 219227.

[10] R. Rees, Two new direct product-type constructions for resolvable group-divisible designs, J.Combin.De, 1 (1993), 15-26. 